\title{
Naive learning and cooperation in network experiments*
}

\author{
Oliver Kirchkamp ${ }^{\dagger} \quad$ Rosemarie Nagel ${ }^{\ddagger}$ \\ 16 January 2006 \\ to appear in Games and Economic Behavior
}

\begin{abstract}
In this paper we study learning and cooperation in repeated prisoners' dilemmas experiments. We compare interaction neighbourhoods of different size and structure, we observe choices under different information conditions, and we estimate parameters of a learning model.

We find that naive imitation, although a driving force in many models of spatial evolution, may be negligible in the experiment. Naive imitation predicts more cooperation in spatial structures than in spaceless ones - regardless whether interaction neighbourhoods have the same or different sizes in both structures. We find that with some interaction neighbourhoods even the opposite may hold.
\end{abstract}

JEL-Classification: C72, C92, D74, D83, H41, R12

Keywords: Imitation, learning, local interaction, heterogeneity of environment, experiments, prisoners' dilemma.

\footnotetext{
${ }^{*}$ We would like to express our thanks to the German DFG (through SFB 303 and SFB 504) and Spain's Ministry of Education under Grants SEC2002-03403 PB98-1076 and SEC2005-08931 and the Barcelona Economics Program of CREA for support. We are grateful to Antonio Cabrales, Jörg Oechsler, Avner Shaked, Nick Vriend and three anonymous referees for their very detailed and helpful comments.

$\dagger$ University of St Andrews, School of Economics and Finance, St Andrews, Fife KY16 9AL, Scotland, UK, email: oliver@kirchkamp.de, phone +44-1334-462440, fax +44-1334-462444.

†niversitat Pompeu Fabra, Dep.of Economics, 132, Balmes, E-08008 Barcelona, email: rosemarie.nagel@upf.edu, phone +34-935-422739, fax +34-935-421746.
} 


\section{Introduction}

How does the structure of interaction affect an evolutionary process? Axelrod (1984, p. 158ff) gives an influential example based on a prisoners' dilemma: In a situation where all players are interacting with all other players in the same way, defectors are always more successful than cooperators and, as a result, cooperation will die out. However, if interaction is local, i.e., players are only interacting in neighbourhoods, cooperation can grow in clusters. As a result, only cooperators on the border of these clusters are exploited by defectors. The local structure protects the emergence of cooperators. Axelrod presents this idea with a specific evolutionary dynamic: players use a copy best rule, i.e. they choose the strategy with the currently highest payoff in their neighbourhood. Nowak and May (1992), Eshel, Samuelson, and Shaked (1998) and several other articles ${ }^{1}$ follow this approach. A simple rule like copy best can apparently explain how cooperation emerges through naive imitation in networks and, thus, opens the door for a host of new and interesting equilibria.

How convincing is such an explanation? In this paper we use experiments to test whether players' behaviour can safely be approximated as sufficiently similar to copy best, and whether players, indeed, are more likely to cooperate if they are in local interaction. We will put emphasis on the assumption of symmetric learning that is implicit in the model of Axelrod and his successors. When players change their strategy, they copy the most successful strategy in their neighbourhood. This strategy can be a player's own strategy or a strategy of a neighbour. Assuming this symmetric treatment of neighbours' and own information may be obvious and innocent in some contexts. E.g. biological evolution does not have the cognitive capabilities to make a distinction between the success of an incumbent species and the success of an invading species. However, evolution of human behaviour may be able to treat own success and the success of neighbours in different ways. Depending on the environment it might be rational to make such a distinction. If agents and neighbours are in the same environment a neighbours' experience is as good as an agent's own experience - there is no reason to value information from different sources differently. In a heterogeneous environment, though, the experience of a neighbour may be specific to a situation that is different from the agent's situation. It might be wrong to draw inference from a neighbour's payoff to one's own success ${ }^{2}$. In such an environment agents should learn relatively more from their own experience and relatively less from the experience of other players.

\footnotetext{
${ }^{1}$ See also Nowak and May (1993), Bonhoeffer, May, and Nowak (1993), Lindgreen and Nordahl (1994), Kirchkamp (2000).

${ }^{2}$ See Kirchkamp (1999).
} 
To control the degree of homogeneity in our experiments, we compare two structures: In one structure agents are located on a circle and interact in overlapping neighbourhoods. This is what we call local interaction or a spatial structure. In such a structure players' environments are not entirely identical. Players may learn from their neighbours, however, their neighbours' success might be due to opponents that are not part of the interaction neighbourhood of the learning players. In the other structure agents are in a homogeneous group where each agent is equally likely to interact with every other agent. This is what we call group interaction or spaceless structure. In this structure all agents face the same interaction partners.

In section 2 we will summarise some arguments in the context of imitation and local interaction which we find helpful to understand our setup. Section 3 presents a theoretical argument based on imitation which suggests more cooperation in a spatial world than in a non-spatial world. Section 4 describes the setup of the experiment. Section 5 presents the experimental results. Section 6 concludes.

\section{Literature and Motivation}

From several other experiments we know that players learn from their own experience and that they also imitate. A classic study that describes how players learn from their own experience is Erev and Roth (1998). Pingle and Day (1996) find that participants of their experiments imitate choices of others to economise decision cost. Offerman and Sonnemans (1998) observe that players imitate beliefs of other players if these are available. Offerman and Sonnemans (1998, p. 571) suggest that own experience might be "more important" for the adaptation of beliefs than naive imitation of others.

What kind of framework should we use in order to study learning and imitation? Here we follow Axelrod's idea and choose a very simple framework, a prisoners' dilemma. This is not the only possible choice. Some recent studies of imitation behaviour use the context of an oligopoly. The oligopoly framework is particularly interesting in the context of learning and imitation since learning and imitation may affect the equilibrium process. Vega-Redondo (1997) presents a theoretical analysis of a Cournot oligopoly and finds that an imitation based evolutionary process converges to the Walras equilibrium which is far away from the Cournot-Nash equilibrium and which is also more competitive. Huck, Normann, and Oechssler (1999) and Offerman, Potters, and Sonnemans (2002) use experiments to show that players do imitate and tend to converge to the Walras equilibrium in oligopolies if information about other players is available. Selten and Ostmann (2001) develop the theoretical concept of an imitation equilibrium which is 
studied in Selten and Apesteguia (2005) with the help of an experiment based on an oligopoly with spatial competition. Selten and Apesteguia find that, indeed, features of the imitation equilibrium describe parts of actual behaviour better than the Cournot Nash concepts. In another oligopoly experiment, however, Bosch-Domènech and Vriend (2003) find imitation not to be a driving force in the experiments and, accordingly, no convergence to the Walras equilibrium.

These experiments help to distinguish among different equilibrium concepts in oligopoly models. However, these experiments also show that the framework of oligopolistic interaction is perhaps not ideally suited to disentangle imitation of others from learning from own experience. The reason is the large strategy space that usually comes with the model of an oligopoly. Players can and will choose many different strategies among a large number of possible quantities. Often players will choose new quantities that have not been tried before. How can we interpret the choice of new quantities as imitation or learning from own experience? Perhaps the chosen strategy was close to one or more successful strategies used by other players or used by the learning player, but how close must a choice be to be qualified as imitation? With so many candidate strategies one needs additional assumptions to relate players' choices to past strategies. ${ }^{3}$

With the prisoners' dilemma we study a game with only two strategies and, thus, reduce the above problem substantially. This game is conceptually close to an oligopoly game, still, with only two strategies it is technically easier to interpret choices as learning. Furthermore, a prisoners' dilemma is not only interesting because it describes the well known dilemma situation. What is useful here are two other properties: firstly, learning and myopic optimisation may call for very different actions in this game, and, as mentioned above, the interaction structure may crucially determine the behaviour of a population. If players copy successful strategies from their neighbours, cooperation may be a stable outcome in prisoners' dilemma games in a locally structured population, but can not be stable in a population without such a structure (see footnote 1 ).

Experiments where players are linked through a network and, thus, are in a heterogeneous situation have been done with coordination games, market games and prisoners'

\footnotetext{
${ }^{3}$ A solution for a related problem is used by Huck, Normann, and Oechssler (2000). In each round the authors determine a best-reply quantity and an imitation quantity. Then they count the number of choices that are within an interval around these two quantities. If we want to use this approach to distinguish between imitating others and learning from own experience we have to deal with the problem that in each round the best strategy is either used by the learning player or used by one of the other players. In each round there is one explanatory observation missing. To impute this missing observation one could assume that players create two polynomial models, one for own experience and the other for others' experience and then maximise given these models to find the missing observations. In this paper we reduce complexity by reducing the strategy space. We may still have to impute some missing values, but we can do this in a much simpler way.
} 
dilemma games. Kosfeld (2004) provides an exhaustive summary of networks experiments. Close to our study are those of Keser, Ehrhart, and Berninghaus (1998), Cassar (2002), and Selten and Apesteguia (2005).

Keser, Ehrhart, and Berninghaus (1998) study how the structure of the network affects selection of Pareto and risk dominant equilibria in coordination games. However, in coordination games we can not distinguish between a player who chooses a strategy as a result of imitating successful neighbours, and a player who chooses a strategy as a result of myopic optimisation. Both motives call for the same action. Since we want to learn more about imitation we have to study a different game.

Cassar (2002) studies coordination games and prisoners' dilemmas. In her experiments with prisoners' dilemmas she finds how perturbations in the structure of a spatial network affects choices. She compares three structures, a local one, a slightly perturbed one (what she calls a small world) and a random network. She finds an interesting non-monotonicity: The slightly perturbed network yields the smallest amount of cooperation.

Selten and Apesteguia (2005) study an oligopoly with a spatially differentiated product. They are, however, not interested in the relation between learning from own experience versus imitation. They do not measure this relationship and they do not vary the heterogeneity of their environment. What they find is that imitation seems to be a relevant factor. What we want to find in this article is how relevant this factor is, as compared to learning from own experience.

While we use space here to model similarity of situations and to allow studying the evolution of strategies, space is also crucial in many economic situations. Restaurants or shops along a street do not compete with the same intensity with all other restaurants or shops. Strategic interaction and imitation of successful strategies may be more important among producers of similar products. Should we, therefore, find more tacit collusion in industries where product space or geographic space is relevant for interaction?

In our experiment groups of players repeatedly play prisoners' dilemmas either within a locally structured neighbourhood (a circle with overlapping neighbourhoods) or within an unstructured (spaceless) group. Players receive information about their neighbours and their own payoffs. We will see that players learn from their own experience. Success of their neighbours, however, does not seem to play a large role. This holds for both structures: the spatial as well as the spaceless one. As a consequence we do not find the higher levels of cooperation in the spatial structure predicted by the theoretical literature under the assumption of learning from neighbours (see footnote 1). Various modifications of our setup do not change this result. 


\section{A simple model based on copy best}

In this section we will sketch a simple and common evolutionary learning process based on copy best ${ }^{4}$ which suggests more cooperation in a spatial environment and less in a non-spatial one.

Let us assume that a a prisoners' dilemma is played in a neighbourhood of $n$ players. Players use the same strategy against all their $n-1$ neighbours. If we call the number of cooperators $n^{C}$ then the payoffs from cooperation and defection, $u^{C}$ and $u^{D}$, are as follows:

$$
\begin{aligned}
& u^{C}=n^{C} \cdot \frac{20}{n-1} \\
& u^{D}=n^{C} \cdot \frac{20}{n-1}+4 .
\end{aligned}
$$

Each cooperator contributes $20 /(n-1)$ points to the payoff of each neighbour, and each defector adds 4 points to the own payoff. Table 1 shows the payoff matrix for a neighbourhood of five.

In a group without a local structure, non-cooperation is always more successful than cooperation, thus, cooperation always dies out. In the upper part of figure 1 we give an example for the copy best dynamics. A group of five players always plays the strategy with the highest average payoff in their neighbourhood (copy best average). With a small probability ( $1 \%$ in this example) players make a mistake and choose the opposite strategy. This is what evolutionary game theorists call a 'mutation'. Average payoffs for $C$ and $D$ in period $t$ are called $u_{t}^{C}$ and $u_{t}^{D}$, respectively. The mutation rate is called $\epsilon$. If all strategies are used in period $t$, and $u_{t}^{C} \neq u_{t}^{D}$, then we can express the probability to play $c$ tomorrow as follows

$$
P\left(c_{t+1}\right)= \begin{cases}1-\epsilon & u_{t}^{C}>u_{t}^{D} \\ \epsilon & u_{t}^{C}<u_{t}^{D}\end{cases}
$$

If $u_{t}^{C}=u_{t}^{D}$ or one strategy was not used in period $t$ then players repeat their choice with probability $1-\epsilon$. In figure 1 time is shown on the horizontal axis and different players on the vertical axis. The upper part of the figure starts in the first period (shown on the left) with 5 cooperating players who choose cooperation until the first mutant arrives. This happens in our example in period 13 where one player mutates and plays $D$. Being now very successful, this player is imitated by all neighbours and from period 14 on everybody plays $D$. Further mutants that appear in later periods do not lead the group back to

\footnotetext{
${ }^{4}$ Similar processes are used e.g. in Nowak and May 1992; 1993, Bonhoeffer, Nowak, and May 1993, Lindgren and Nordahl 1994, Eshel, Samuelson, and Shaked 1998, Kirchkamp 2000.
} 
Figure 1: Simulated learning.

copy best average or copy best max imitation in a group:

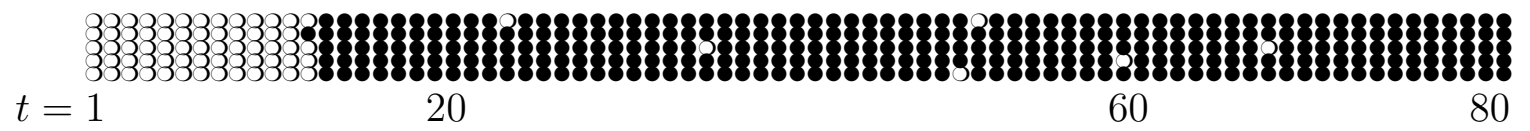

copy best average imitation in a circle:

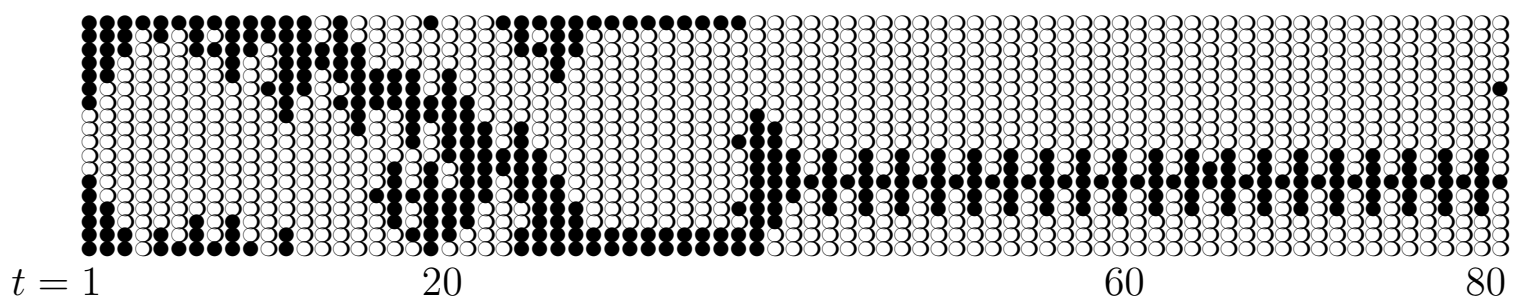

copy best max imitation in a circle:

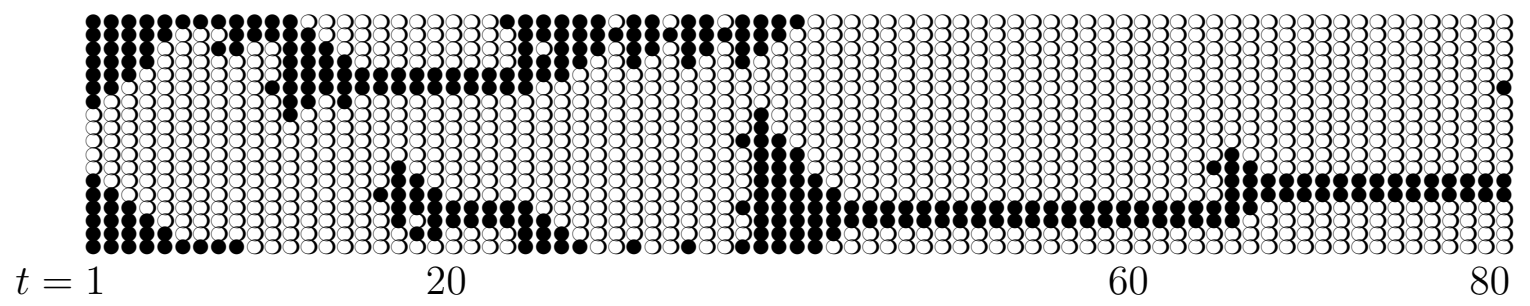

$\circ=C, \bullet=D$. Time is shown on the horizontal axis, different players are shown on the vertical axis.

The first mutant $D$ makes cooperation disappear completely in groups. Cooperation in circles, however, persists despite mutant $D$ s.

(The mutation rate is $1 \%$, the imitation and interaction radius is 2 , as in the experiment. Simulations starts with 5 cooperators in the first period.)

cooperation. ${ }^{5}$

In a spatial setting and with similar imitation dynamics (see footnote 1) however, cooperation is protected through space and may survive. ${ }^{6}$ Let us assume that player 2 from table 2 knows his own payoff from playing $D$, which is 14 , but also the payoff from his two $D$-playing neighbours, 9 and 4 . The average payoff of playing $D$ is, hence, 9 . The two $C$-playing neighbours of this player have a payoff of 15 and 10, on average, hence, 12.5. If player 2 copies the strategy with the highest average payoff then player 2 will choose $C$ in the next period-thus, cooperation will grow. ${ }^{7}$

\footnotetext{
${ }^{5}$ The only way to move a population where everybody plays $D$ back to cooperation is a simultaneous mutation of all five players. With independent mutations this is not very likely. And even if it happens, cooperation will not last for long since the first single mutant leads the population back to $D$. As a result the population will spend most of the time in a state where most of them play $D$.

${ }^{6}$ With myopic optimisation (Ellison, 1993) players would obviously never cooperate.

${ }^{7}$ Once the cluster of $D$ s becomes small the payoff of the remaining $D$ s grows and the process stops
} 
Table 1: Payoff Matrix

\begin{tabular}{|c|c|c|c|c|}
\hline \multicolumn{5}{|c|}{ Own payoff: } \\
\hline own & number of & $\begin{array}{l}\text { neigh } \\
\text { group }\end{array}$ & & choosing $C$ \\
\hline action & 0 & 2 & 3 & 4 \\
\hline$C$ & 0 & 10 & 15 & 20 \\
\hline $\bar{D}$ & 4 & $\overline{14}$ & 19 & 24 \\
\hline
\end{tabular}

Table 2: Example of a neighbourhood of $C$ s and $D$ s

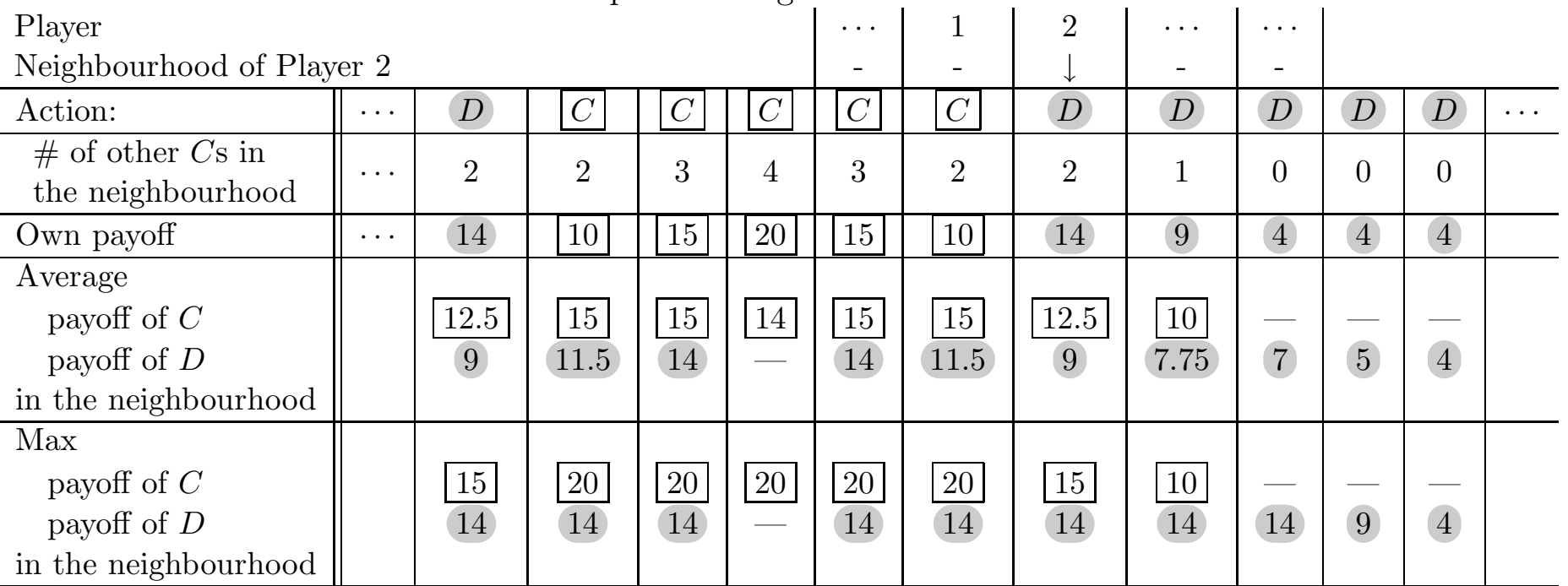


In our example (see the middle part of figure 1) cooperation grows from the initial configuration of only five $C$ s and is not much affected by mutants.

In describing the above dynamics we used the rule copy best average payoff (see the literature given in footnote 4). A similar dynamics is copy best max which we obtain if in equation (3) the variables $u_{t}^{C}$ and $u_{t}^{D}$ denote maximal and not average payoffs in the neighbourhood. The bottom part of figure 1 gives an example for the copy best max dynamics. An example for the calculation of payoffs is given at the end of table 2. As with copy best average, also with copy best max a small cluster of cooperative players turns out to be successful and grows through imitation.

We should note that neither copy best max nor copy best average distinguish between a players' own experience and his neighbours' experience. This is expressed in the following hypothesis:

Hypothesis SYM-LEARN: Players learn as much from his neighbours' experience as from their own.

It is not obvious that hypothesis SYM-LEARN should hold. In a spatial structure players' environments are not identical. Making no distinction between own experience and a neighbours' experience may be suboptimal. ${ }^{8}$ We summarise this in the following hypothesis:

Hypothesis ASYM-LEARN: Players learn relatively more from their own experience and less from their neighbours' experience the more local their interaction structure is.

If hypothesis SYM-LEARN holds, then we should, following the argument sketched in section 3 and discussed in detail in the literature (see footnote 4), expect the following:

Hypothesis COOP-SPACE: We find more cooperation in populations with a spatial structure than in populations without such a structure.

If, however, learning is not symmetric and instead ASYM-LEARN holds, the forces of imitation are weaker. Imitation of neighbours is, as we have seen in the example above, a major driving force behind the survival of cooperation in a spatially structured population. A player who looks only at his own payoff in a prisoners' dilemma quickly learns that defection gives a higher payoff-regardless whether this player is learning in a spatial or a spaceless structure. We might then find the following:

or enters a cycle. With standard imitation processes stable equilibria are often reached when clusters of successful $C$ s are separated by small clusters of equally successful $D$ s.

${ }^{8}$ See Kirchkamp (1999). 
Hypothesis NOCOOP-SPACE Levels of cooperation are not higher in a spatial structure.

Other experiments have shown (see e.g. Fox and Guyer, 1977) that cooperation is easier to establish in a small neighbourhood:

Hypothesis COOP-SMALL Levels of cooperation are larger in small neighbourhoods.

\section{The experimental setup}

In this paper we describe results from seven different treatments which are based on 44 sessions run in Barcelona and Mannheim, involving 423 participants $^{9}$. Interaction in the experiment was computerised and anonymous. The number of participants in the lab was always larger than the size of a neighbourhood, so that participants could not identify their neighbourhood. A list of these sessions is given in appendix A.

During a session players always interact with the same neighbours. Sessions last for 80 periods. In each period participants play a prisoners' dilemma against all members of their neighbourhood/group. Payoffs are given by equations (1) and (2). We compare three interaction structures:

circles: This structure is shown in the left part of figure 2. Players are indirectly connected through overlapping neighbourhoods of 5 players. In the experiment participants are randomly seated in front of computer terminals that are networked to create the neighbourhood structure. Each player interacts in each round with two neighbours to the left and two neighbours to the right. Player $x_{0}$ in the figure is in interaction with $x_{1}, x_{2}$, and $y_{1}, y_{2}$. Player $x_{2}$ is in interaction with $x_{3}, x_{4}$, and $x_{1}, x_{0}$. Players see this structure on the screen. ${ }^{10}$

groups: This structure is shown in the middle of figure 2. Players are either directly connected or not connected at all. As in the circle treatment each player has four neighbours. Players see this structure on the screen.

\footnotetext{
${ }^{9}$ Students of the UPF in Barcelona and Universität Mannheim respectively.

${ }^{10}$ The circles in figure 2 show a simplified picture of the neighbourhood. Not all players that interact are connected with lines. E.g. $x_{0}$ has four neighours. In the graph there is a line from $x_{0}$ to $x_{1}$ and $y_{1}$ but no direct line from $x_{0}$ to $x_{2}$ and to $x_{4}$ (though $x_{1}, x_{2}, y_{1}$, and $y_{2}$ are highlighted). When we planned the experiment we compared various representations and showed them to students. We found the representation in figure 2 more efficient than the one which was cluttered with the entire set of connections. Furthermore, at the beginning of the experiment each subject participates in a quiz. Participants have to answer several questions regarding the structure of the neighbourhood. Those who gave the wrong answers would get feedback and extra help on the screen.
} 
Figure 2: Neighbourhoods neighbourhoods of 5

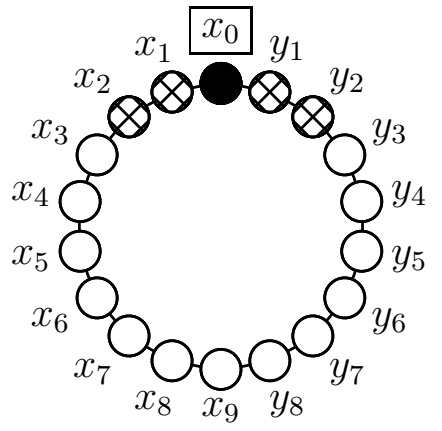

Circle: spatial interaction of players through overlapping neighbourhoods

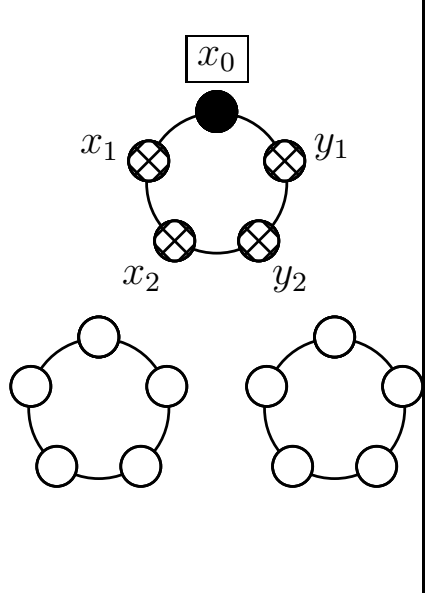

neighbourhoods of 10
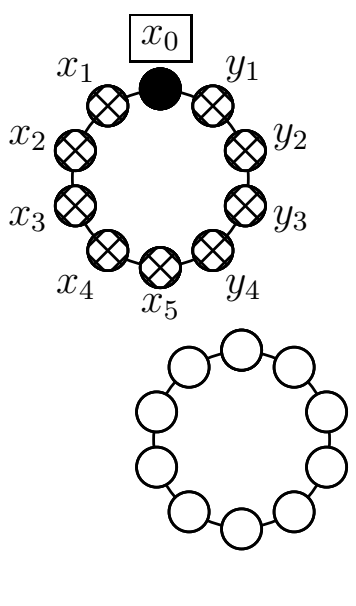

Groups: non-spatial interaction, all players are either in the same neighbourhood, or do not interact at all.

groups with a larger neighbourhood: An example for this structure is shown in the right part of figure 2. As in the group treatment, all players are either directly connected or not connected at all, but the neighbourhood is larger than in the two other treatments and has a size between 8 and 10 .

Following the copy best average or the copy best max model discussed above we should expect almost no cooperation in the two group structures, and we should expect cooperation in the circle structure.

We will study learning in two different information settings. One is closer to the copy best average learning rule from section 3 , the other is closer to the copy best max rule and gives players some insight into the strategic structure of the game.

no detailed information: In this treatment feedback is given as shown in the upper part of table 3. Players see their own action and payoff, as well as average payoffs $u^{C}$ and $u^{D}$ with the two strategies in their neighbourhood (including their own payoff). Players do not know the payoff matrix of the game (table 1) but they have all the information they need for copy best average.

detailed information: In this treatment feedback is given as shown in the lower part of table 3. Players see their own action and payoff, as well as all actions and payoffs in their neighbourhood. Payoffs and strategies of the neighbours are ordered by payoff, i.e. information about the same player may appear in different columns at different times. Furthermore players see the payoff matrix (table 1). 
Table 3: Feedback given in the different treatments

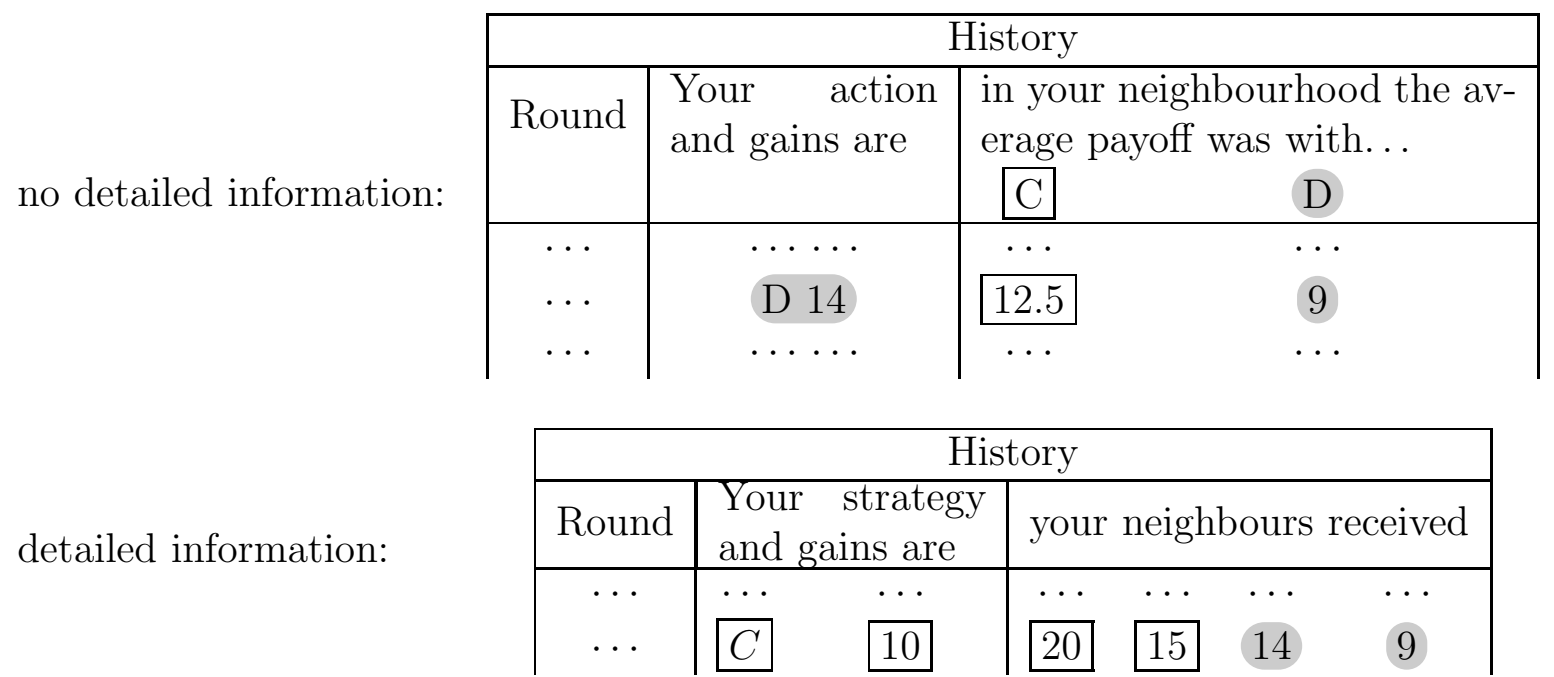

In the experiment strategies were called A and B. In some sessions A was the cooperative strategy, in others B. This was randomly determined before the experiment. Payoffs of $C$ s are shown in a box, payoffs of $D$ s are shown in gray. In the experiment we used different background colours for the different strategies. In the treatment with detailed information payoffs and strategies of the neighbours are ordered by payoff, i.e. information about the same player may appear in different columns.

Thus, in the detailed information treatment players have all the information they need to apply a rule like copy best max. In addition they have some information that is not relevant for copy best max but that might be interesting for a strategic analysis of the game.

In this series of experiments we do not study setups with even more detailed information since we want to concentrate on the copy best average and copy best max learning rules. These two learning rules do not distinguish between neighbours in different positions. Therefore, in this experiment, we do not differenciate between payoffs of neighbours which are more or less close.

\section{$5 \quad$ Results}

We will first study stage game behaviour. Anticipating our results, we will find no support for hypothesis COOP-SPACE. Then we will relate this observation to learning. We will see that imitation of neighbours is only a weak force. Players' behaviour is much more driven by their own experience than by their neighbours' experience. This contradicts hypothesis SYM-LEARN, but is in line with hypothesis ASYM-LEARN. The players' 
Figure 3: Frequency of cooperative players in circles and groups over time
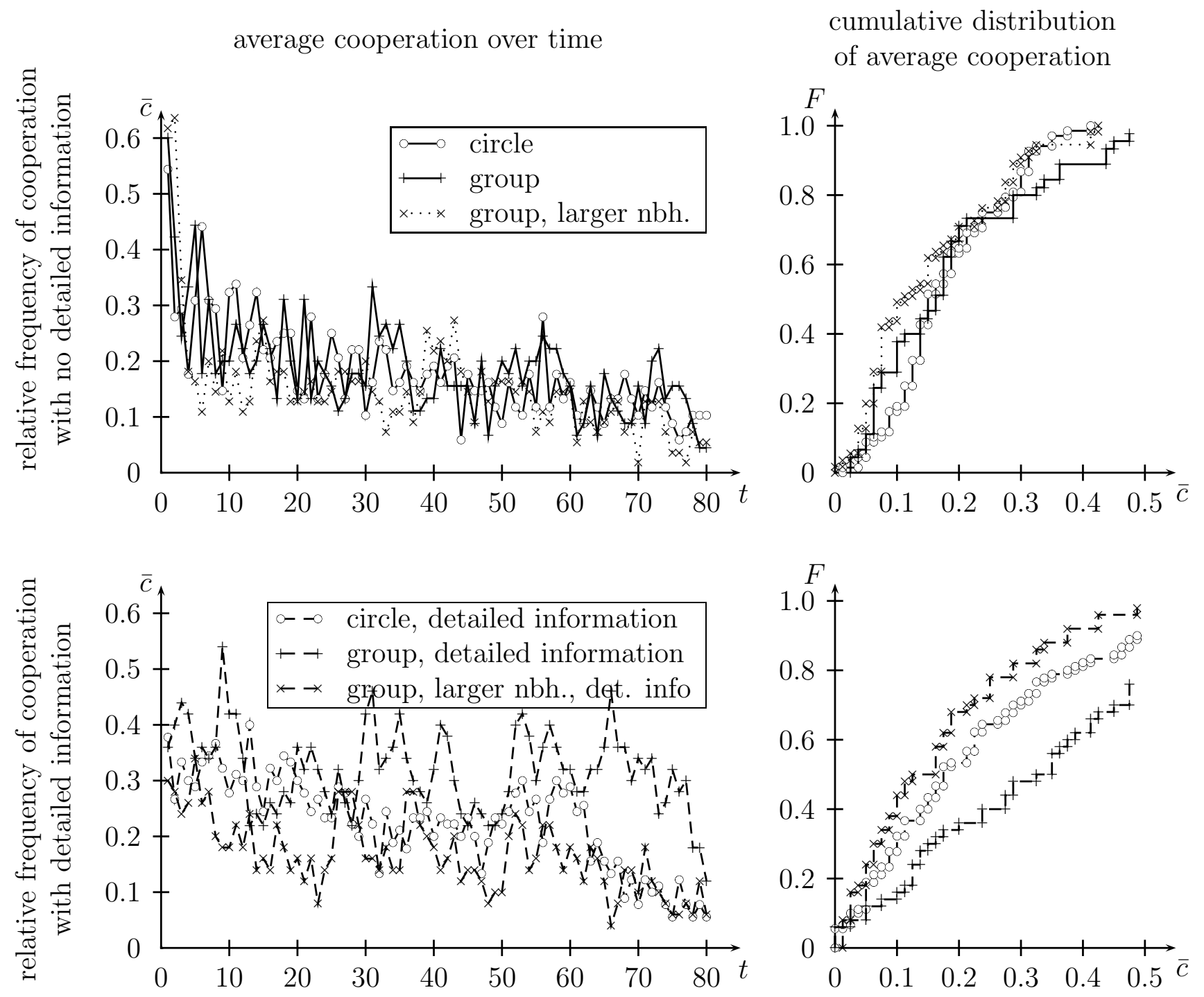

actions over time are shown in appendix B.1 and B.2.

\subsection{Stage game behaviour}

In figure 3 we show relative frequencies of cooperation $\bar{c}$ for the different treatments. The top graphs show treatments without detailed information, the bottom graphs show treatments with detailed information. The left graphs show relative frequencies of cooperation over time, the right graphs cumulative distributions of relative frequencies of cooperation for the different individuals.

Let us first look at the behaviour in groups of different sizes. Following hypothesis COOP-SMALL we should expect less cooperation in groups with a larger neighbourhood. 
Table 4: Less cooperation in large neighbourhoods

\begin{tabular}{l|llllll|} 
& $n$ & $\beta$ & $t$ & $P_{<t}$ & $z$ & $P_{<z}$ \\
\hline no detailed information & 15 & -.0348 & -1.39 & 0.093 & -1.061 & 0.144 \\
detailed information & 15 & -.1497 & -4.14 & 0.000 & -2.694 & 0.004
\end{tabular}

The table shows the result of estimating (for the group experiments only) $c=\beta_{0}+\beta d_{\text {large }}$ where $d_{\text {large }}=1$ in large neighbourhoods and zero otherwise. A negative $\beta$ means that there is less cooperation in large neighbourhoods.

Table 5: Different levels of cooperation in circles and groups

\begin{tabular}{l|cccccc|} 
& $n$ & $\beta$ & $t$ & $P_{>t}$ & $z$ & $P_{>z}$ \\
\hline no detailed information & & & & & & \\
circle vs. groups & 18 & -.0081 & -.365 & 0.640 & -0.221 & 0.587 \\
circle vs. groups with large nbhds. & 15 & .0268 & 1.89 & 0.040 & 1.650 & 0.049 \\
\hline detailed information & & & & & & \\
circle vs. groups & 15 & -.0913 & -2.89 & 0.994 & -1.715 & 0.957 \\
circle vs. groups with large nbhds. & 10 & .0584 & 2.33 & 0.022 & 1.776 & 0.038
\end{tabular}

The table shows the result of estimating $c=\beta_{0}+\beta d_{\text {circle }}$ where $d_{\text {circle }}=1$ in circles and zero otherwise. A positive $\beta$ means that there is more cooperation in circles, a negative $\beta$ means that there is less cooperation in circles.

We test this with the help of a one-sided $t$ test $^{11}$ and a one-sided Wilcoxon rank sum test. ${ }^{12}$ Results are shown in table 4 . In both information treatments we find less cooperation in large neighbourhoods $(\beta<0)$. This is significant in the detailed information treatment. In the no detailed information treatment the difference is only weakly significant and only for the $t$-test. Thus, in particular in the detailed information condition we find support for hypothesis COOP-SMALL.

Let us now come to the effect of the interaction structure. Following hypothesis COOP-SPACE there should be more cooperation in the spatial structure (in circles) than in groups - regardless what the size of the neighbourhood of the groups actually is. Again, we present results of a one-sided $t$-test and a one-sided Wilcoxon rank sum test. Results are shown in table 5. If we use neighbourhoods of the same size as a basis of our

\footnotetext{
${ }^{11}$ When calculating levels of standard deviations and levels of significance we have to take into account that observations within our experimental sessions may be correlated. We can safely assume that covariances of observations from different sessions are zero. Covariances of observations from the same experiment are replaced by the appropriate product of the residuals (Rogers, 1993). We will use this approach throughout the paper to calculate standard errors.

${ }^{12}$ The reason to present both parametric and non-parametric tests here is the following: The parametric test has more power. Since we want to show that in some cases where a difference is expected no significant difference can be found, we should use the strongest possible test. Indeed, the assumption of normality does not seem to be far fetched when we look at the cumulative distributions in figure 3 .
} 
Figure 4: The structure of circles with some computerised players

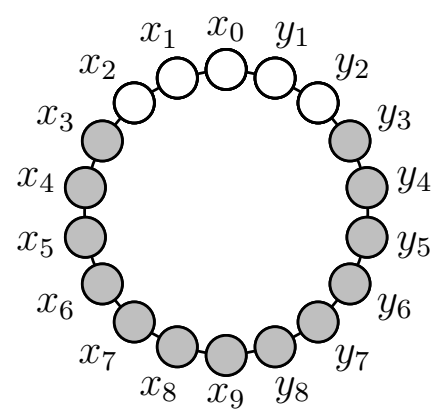

The five white dots indicate the position of computerised players that always play $C$. The remaining dots indicate the position of the human players. Neither the position nor the strategy of the computerised players was know to the human players.

comparison (the circle vs. groups case in table 5) then we find no support for hypothesis COOP-SPACE.

Only when we compare circles with a neighbourhood of 5 with groups of a neighbourhood of 8 to 10 , we find a positive $\beta$ and a significant difference.

\subsection{A treatment with some computerised players}

In section 3 we explained how imitation of successful neighbours supports cooperation in a spatial environment. This argument relies on the existence of an initial cluster of cooperators of sufficient size - with our payoff matrix five neighbouring cooperators are sufficient to ensure imitation. An evolutionary game theorist would be confident that in the long run and through mutations such a cluster will appear eventually. In our experiments suitable clusters do appear, but they are not imitated. Perhaps, if these clusters were more persistent then imitation of neighbours would start and cooperation would grow in the local interaction structure.

To test this and to give imitation the best possible chance we introduce a cluster of five computerised players into the circle. In figure 4 players $x_{2}, x_{1}, x_{0}, y_{1}, y_{2}$ are played by the computer and cooperate in every period. The other participants are humans who obtain the same information as in the treatment with no detailed information. Furthermore they are told that "... In addition to the players that are in a room, five players follow a computerised strategy. Up to two of your neighbours may belong to these players...". Participants were not told what strategy the computerised players would follow. Details of the instructions can be found in appendix C. The detailed behaviour of the human 
Figure 5: Cooperation depending on the distance to the computerised players

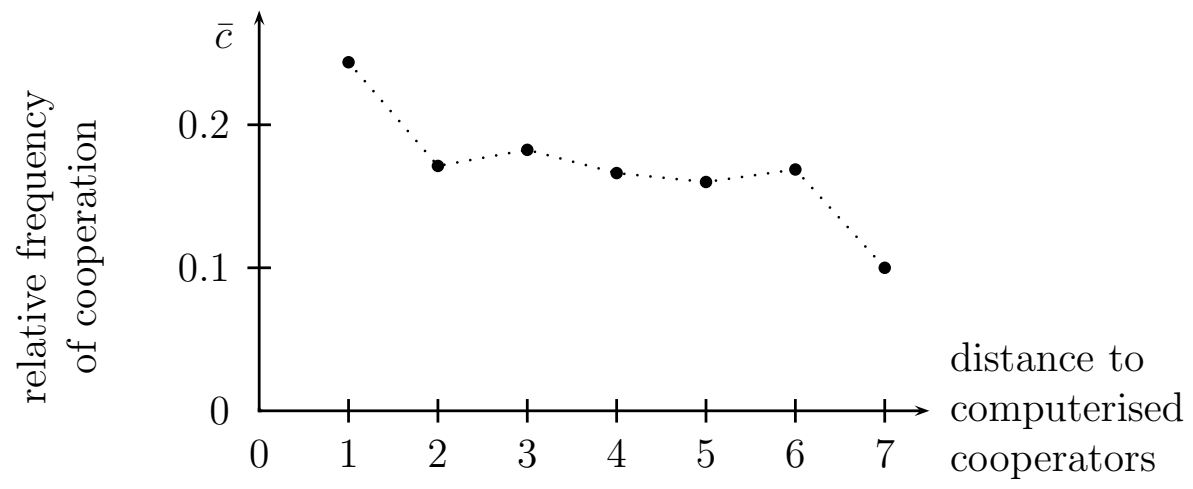

Table 6: Increase in cooperativeness due to computerised cooperators

\begin{tabular}{l|cccccc|} 
& $n$ & $\beta$ & $t$ & $P_{>t}$ & $z$ & $P_{>z}$ \\
\hline $\begin{array}{l}\text { immediate neighbours of } \\
\text { of computerised players }\end{array}$ & 14 & .0366 & .622 & 0.272 & 0.333 & 0.369 \\
all players & 14 & -.0314 & -2.38 & 0.983 & -1.533 & 0.937
\end{tabular}

The table shows the result of estimating $c=\beta_{0}+\beta d_{\text {comp }}$ where $d_{\text {comp }}=1$ in circles with computerised players and zero otherwise. A positive $\beta$ means that there is more cooperation with computerised cooperators, a negative $\beta$ means that there is less cooperation with computerised cooperators. Test are against $d_{\text {comp }} \leq 0$.

players is shown in appendix B.6.

Figure 5 shows how the frequency of cooperation depends on the distance to the computerised players. We see that a good example helps a little - at least if it is close enough. Players with a smaller distance to the computerised players cooperate significantly more. ${ }^{13}$ However, the effect is not very strong. Figure 6 shows the development of cooperation and the cumulative distribution of the individual frequency of cooperation in the baseline treatment and in circles with computerised players. We see that levels of cooperation are very close to each other. A formal comparison can be found in table 6. Players who are located immediately next to a computerised cooperator cooperate more than players from the treatment without computerised cooperators, though the difference is not significant. The average player in the treatment with computerised players cooperates even slightly less than those without.

To summarise: Even introducing a 'permanent good example' does not increase significantly the frequency of cooperation in circles. If we find no support for hypothesis

\footnotetext{
${ }^{13}$ A Cuzick-Altman test finds $z=-2.10, P>|z|=0.036$.
} 
Figure 6: Cooperation in circles with some computerised players average cooperation over time cumulative distribution (no detailed information) of average cooperation
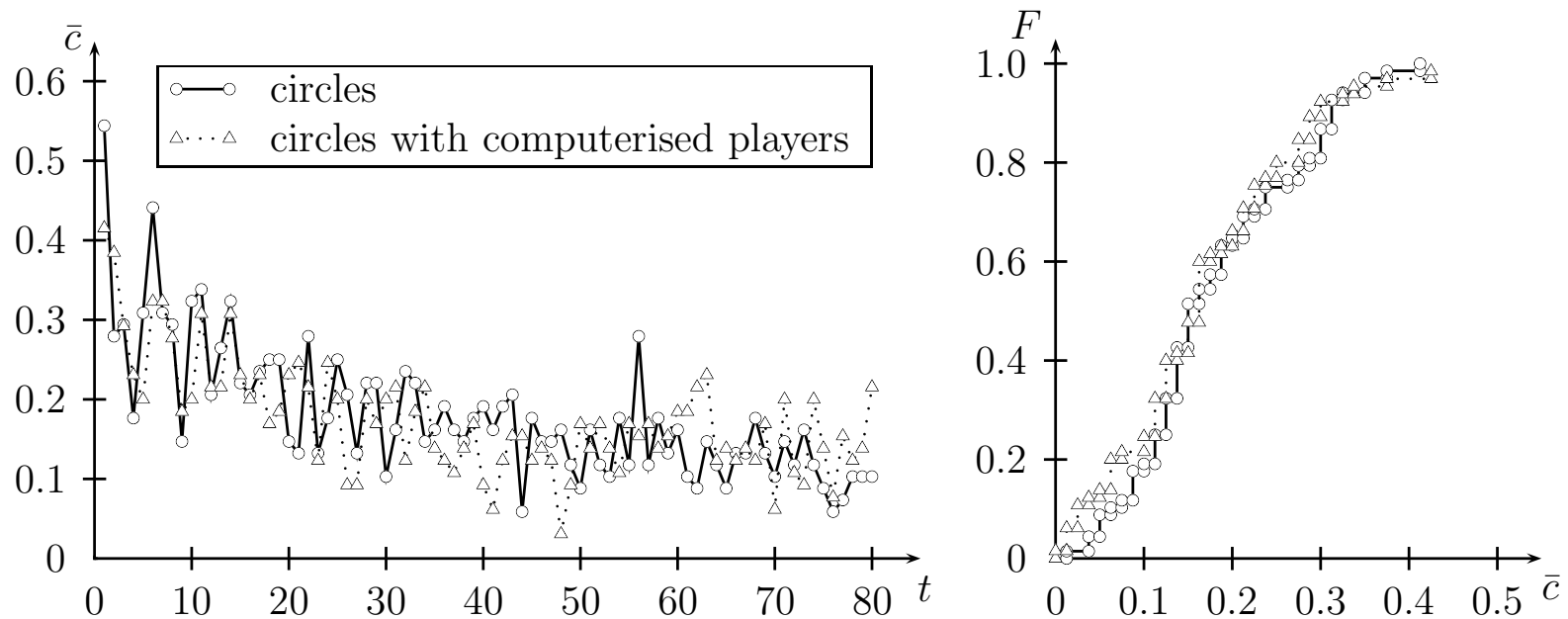

The left graphs show the relative frequency of cooperation for each period in each treatment. The right graphs show the cumulative average frequency (averages for each player) for the different treatments.

COOP-SPACE the reason can not be that there are not sufficiently many cooperative players to imitate. There must be another cause - we suspect that players do not imitate at all. This is what we will test in the next section.

\subsection{Learning}

In this section we will investigate hypothesis SYM-LEARN and ASYM-LEARN. We use a logit model to describe discrete choices between two alternatives, $C$ and $D$. This allows us to draw inference from choices to the learning process. Own payoffs from $C$ and $D$ at time $t$ will be called $u_{t}^{c, \text { own }}$ and $u_{t}^{d \text {,own }}$, respectively. In a similar way $u_{t}^{c \text {,other }}$ and $u_{t}^{d \text {,other }}$ describe average payoffs of the other players with the two strategies. If a given action $s \in\{C, D\}$ was not chosen at time $t$ by a player or in the neighbourhood ( $i \in\{$ own, other $\}$ ) then we recursively use $u_{t}^{s, i}:=u_{t-1}^{s, i}$ until we reach a period where $s$ was chosen. One could say that $u_{t}^{c, \text { own }}, u_{t}^{d \text {,own }}, u_{t}^{c \text {,other }}, u_{t}^{d \text {,other }}$ represent the most recent information players have about the success of their strategies at time $t$. In line with equation (3) we use differences in payoffs of $C$ and $D$ as explanatory variables of our model. $\Delta_{t}^{\text {own }}:=u_{t}^{c, \text { own }}-u_{t}^{d \text {,own }}$ is the difference between payoff from cooperation and payoff from non cooperation as experienced by the player in period $t . \Delta_{t}^{\text {other }}:=u_{t}^{c \text {,other }}-u_{t}^{d \text {,other }}$ is the difference between payoff from cooperation and payoff from non cooperation as experienced by player's neighbours in 
Table 7: Estimation of equation (4)

\begin{tabular}{l|cc} 
& GEE & logit \\
\hline$c_{t}$ & 2.601 & 1.793 \\
& $(83.10)^{* *}$ & $(19.50)^{* *}$ \\
\hline$\Delta^{\text {own }}$ & 0.054 & 0.059 \\
& $(16.26)^{* *}$ & $(6.71)^{* *}$ \\
\hline $\bar{\Delta}^{\text {own }}$ & 0.002 & 0.004 \\
& $(0.43)$ & $(0.20)$ \\
\hline$\Delta^{\text {other }}$ & 0.009 & 0.011 \\
& $(4.05)^{* *}$ & $(2.87)^{* *}$ \\
\hline $\bar{\Delta}^{\text {other }}$ & 0.001 & 0.001 \\
& $(0.21)$ & $(0.16)$ \\
\hline constant & -1.811 & -1.536 \\
& $(66.25)^{* *}$ & $(19.60)^{* *}$ \\
\hline Observations & 30599 & 30599
\end{tabular}

$\Delta$ denotes the current payoff difference between the two actions, $\bar{\Delta}$ denotes average payoff difference. Estimated coefficients, thus, denote propensities to learn from own and other current or average payoffs. Absolute value of z-statistics in parentheses, * significant at $5 \%$; ** significant at $1 \%$

period $t .^{14}$

We will also look at average payoffs of the two strategies over all periods until period $t$. We will call average payoffs $\bar{u}_{t}^{c \text {,own }}, \bar{u}_{t}^{d \text {,own }}, \bar{u}_{t}^{c \text {,other }}, \bar{u}_{t}^{d \text {,other }}$. Differences between average payoffs are called $\bar{\Delta}_{t}^{\text {own }}$ and $\bar{\Delta}_{t}^{\text {other }}$.

To allow for some inertia we include the current choice $c_{t}$ which we code as 1 if the player cooperates today, and 0 otherwise. We will first estimate

$$
P\left(c_{t+1}\right)=\mathcal{L}\left(\beta_{0}+\beta_{c} c_{t}+\beta^{\text {own }} \Delta^{\text {own }}+\beta^{\text {other }} \Delta^{\text {other }}+\gamma^{\text {own }} \bar{\Delta}^{\text {own }}+\gamma^{\text {other }} \bar{\Delta}^{\text {other }}\right)
$$

where $\mathcal{L}(x)=e^{x} /\left(1+e^{x}\right), c_{t+1}$ is 1 if a player cooperates tomorrow, and 0 otherwise. Table 7 compares results of two estimation models for equation (4), a GEE and a logit model. The GEE model takes the autocorrelation of payoffs and choices into account and models equation (4) as an AR(1) process. The logit model disregards the autocorrelation. We see that estimated coefficients are very similar for the two methods. Since the GEE estimator does not always converge for subsets of our data we will present results for subsamples in the following only for the logit model. ${ }^{15}$

\footnotetext{
${ }^{14}$ Using counterfactual payoffs is not possible here since then the difference $\Delta^{\text {own }}$ would be a constant which would make it impossible to estimate any effect.

${ }^{15}$ In estimating equation (4) we make the implicit assumption that learning behaviour is constant over time. In Kirchkamp and Nagel (2005) we test this and find that changes over time do not follow an
} 
Table 8: Estimation of equation (5)

\begin{tabular}{|c|c|c|c|c|c|c|c|c|c|}
\hline & \multirow[t]{2}{*}{$\begin{array}{c}\text { GEE } \\
\text { all }\end{array}$} & \multirow[t]{2}{*}{ all } & \multicolumn{3}{|c|}{ circle } & \multicolumn{2}{|c|}{ groups } & \multicolumn{2}{|c|}{ large groups } \\
\hline & & & & $\begin{array}{l}\text { detailed } \\
\text { info }\end{array}$ & $\begin{array}{c}\text { computerised } \\
\text { cooperators }\end{array}$ & & $\begin{array}{l}\text { detailed } \\
\text { info }\end{array}$ & & $\begin{array}{c}\text { detailed } \\
\text { info }\end{array}$ \\
\hline$c_{t}$ & $\begin{array}{c}2.601 \\
(83.70)^{* *}\end{array}$ & $\begin{array}{c}1.793 \\
(19.28)^{* *}\end{array}$ & $\begin{array}{c}1.137 \\
(7.89)^{* *}\end{array}$ & $\begin{array}{c}1.930 \\
(18.73)^{* *}\end{array}$ & $\begin{array}{c}1.238 \\
(5.37)^{* *}\end{array}$ & $\begin{array}{c}1.925 \\
(9.39) * *\end{array}$ & $\begin{array}{c}2.035 \\
(17.52)^{* *}\end{array}$ & $\begin{array}{c}1.910 \\
(14.44)^{* *}\end{array}$ & $\begin{array}{c}2.031 \\
(8.64)^{* *}\end{array}$ \\
\hline$\Delta^{\text {own }}$ & $\begin{array}{c}0.054 \\
(18.59)^{* *}\end{array}$ & $\begin{array}{c}0.061 \\
(8.90)^{* *}\end{array}$ & $\begin{array}{c}0.080 \\
(3.76)^{* *}\end{array}$ & $\begin{array}{c}0.062 \\
(4.49)^{* *}\end{array}$ & $\begin{array}{c}0.119 \\
(9.76)^{* *}\end{array}$ & $\begin{array}{c}0.049 \\
(2.45)^{*}\end{array}$ & $\begin{array}{c}0.033 \\
(2.96)^{* *}\end{array}$ & $\begin{array}{c}0.056 \\
(2.75)^{* *}\end{array}$ & $\begin{array}{c}0.031 \\
(1.20)\end{array}$ \\
\hline$\Delta^{\text {other }}$ & $\begin{array}{c}0.010 \\
(9.49)^{* *}\end{array}$ & $\begin{array}{c}0.012 \\
(5.57)^{* *}\end{array}$ & $\begin{array}{l}0.049 \\
(1.72)\end{array}$ & $\begin{array}{l}0.021 \\
(1.27)\end{array}$ & $\begin{array}{l}0.018 \\
(1.59)\end{array}$ & $\begin{array}{l}0.059 \\
(1.64)\end{array}$ & $\begin{array}{c}-0.089 \\
(2.93)^{* *}\end{array}$ & $\begin{array}{l}0.004 \\
(1.14)\end{array}$ & $\begin{array}{l}0.004 \\
(0.71)\end{array}$ \\
\hline Constant & $\begin{array}{c}-1.816 \\
(74.72)^{* *}\end{array}$ & $\begin{array}{c}-1.546 \\
(31.79)^{* *}\end{array}$ & $\begin{array}{c}-1.306 \\
(16.42)^{* *}\end{array}$ & $\begin{array}{c}-1.471 \\
(11.70)^{* *}\end{array}$ & $\begin{array}{c}-1.208 \\
(12.41)^{* *}\end{array}$ & $\begin{array}{c}-1.492 \\
(8.80)^{* *}\end{array}$ & $\begin{array}{c}-2.064 \\
(9.06)^{* *}\end{array}$ & $\begin{array}{c}-1.943 \\
(10.11)^{* *}\end{array}$ & $\begin{array}{c}-1.926 \\
(4.54)^{* *}\end{array}$ \\
\hline Observations & 30599 & 30599 & 5214 & 6386 & 4343 & 3408 & 3526 & 4041 & 3681 \\
\hline
\end{tabular}

Absolute value of z-statistics in parentheses, ${ }^{*}$ significant at $5 \%$; ** significant at $1 \%$. When calculating levels of standard deviations and levels of significance we take into account that observations within any of our sessions may be correlated (see footnote 11).

We also see that coefficients for $\bar{\Delta}^{\text {own }}$ and $\bar{\Delta}^{\text {other }}$ are close to zero and not significant at all. We will therefore concentrate on $\Delta^{\text {own }}$ and $\Delta^{\text {other }}$ in the following and estimate a simplified version of equation (4).

$$
P\left(c_{t+1}\right)=\mathcal{L}\left(\beta_{0}+\beta_{c} c_{t}+\beta^{\text {own }} \Delta^{\text {own }}+\beta^{\text {other }} \Delta^{\text {other }}\right)
$$

With a learning rule like copy best we should expect the coefficient of $\Delta^{\text {other }}$ to be larger than the coefficient of $\Delta^{\text {own }}{ }^{16}$

Table 8 presents estimation results for equation (5). The two leftmost columns compare the GEE with the logit estimation. The coefficients of $\Delta^{\text {own }}$ and $\Delta^{\text {other }}$ are similar to the ones estimated for equation (4) in table 7 , so ignoring average payoffs did not affect the estimation results too much. The columns further to the right show estimation results for the different treatments. We should note two things:

- With only one exception the coefficient of $\Delta^{\text {own }}$ is always larger (and not smaller) than the coefficient of $\Delta^{\text {other }}{ }^{17}$ We can, thus, reject hypothesis SYM-LEARN and support hypothesis ASYM-LEARN.

- Coefficients are always smaller in the treatments with detailed information. In one case the coefficient is even negative. Furthermore, the $c_{t}$ coefficient is always larger in the treatments with detailed information. We presume that when detailed information is available imitation becomes less important and strategic considerations

obvious pattern. All major results that we will show below seem to hold during the whole experiment. Trends, if they can be found at all, are weak and not significant.

${ }^{16}$ To show this we have done extensive Monte Carlo studies with GEE and logit estimations of equation (4) for simulated populations where all players follow copy best. Results are documented in an extended version of this paper (Kirchkamp and Nagel, 2005).

${ }^{17}$ For all treatments and the logit method we find $\chi^{2}=44.0, P_{>\chi^{2}}=0.0000$. 
have more influence.

\section{Conclusion}

The aim of this paper is to better understand how players learn and how their learning behaviour depends on the heterogeneity of their environment. We concentrated on copy best learning, which is a common model of learning in the literature on local interaction. We have seen that learning of human players, in particular in heterogeneous structures, does not fit copy best very well. Players do imitate others sometimes, but they seem to learn primarily from their own experience.

We think that this is a worthwhile contribution to the literature that builds upon imitation in local interaction models to explain cooperation. This literature explains very elegantly how local interaction supports cooperation in an evolutionary context. Regardless whether interaction neighbourhoods have the same or different sizes in both structures we should always find more cooperation in the local interaction structure. We find that at least with interaction neighbourhoods of similar sizes even the opposite may hold. Survival of cooperation in a spatial structure depends substantially on naive imitation of others. If, as we find in our experiments, imitation plays only a minor role in particular in spatial settings, cooperation breaks down.

We also find that the available information affects the amount of imitation in an intuitive way. When more information is available players rely less on imitation. Given that in other games imitation is not much affected by information (see Bosch-Domènech and Vriend, 2003, though they find that other elements of behaviour are affected by complexity) complexity of the game itself might be a moderating factor. In the fairly complex game of Bosch and Vriend players might overlook information altogether, always relying on a certain amount of imitation. In simpler games, like the prisoners' dilemma, information, if available, may have some impact and may displace imitation.

\section{References}

Axelrod, R., 1984, The evolution of cooperation. Basic Books, New York.

Bonhoeffer, S., R. M. May, and M. A. Nowak, 1993, More Spatial Games, International Journal of Bifurcation and Chaos, 4, 33-56.

Bosch-Domènech, A., and N. J. Vriend, 2003, Imitation of successful behavior in cournot markets, The Economic Journal, pp. 495-524. 
Cassar, A., 2002, Coordination and Cooperation in Local, Random and Small World Networks: Experimental Evidence, in Proceedings of the 2002 North American Summer Meetings of the Econometric Society: Game Theory, ed. by D. K. Levine, W. Zame, L. Ausubel, P.-A. Chiappori, B. Ellickson, A. Rubinstein, and L. Samuelson.

Ellison, G., 1993, Learning, Local Interaction, and Coordination, Econometrica, 61, 10471071.

Erev, I., and A. E. Roth, 1998, Predicting How People Play Games: Reinforcement Learning in Experimental Games with Unique, Mixed Strategy Equilibria, American Economic Review, 88(4), 848-81.

Eshel, I., L. Samuelson, and A. Shaked, 1998, Altruists, Egoists, and Hooligans in a Local Interaction Model, The American Economic Review, 88, 157-179.

Fox, J., and M. Guyer, 1977, Group Size and Other's Strategy in an N-Person Game, Journal of Conflict Resolution, 21(2), 323-338.

Huck, S., H.-T. Normann, and J. Oechssler, 1999, Learning in Cournot Oligopoly - An Experiment, The Economic Journal, 109, C80-C95.

— , 2000, Does information about competitors' actions increase or decrease competition in experimental oligopoly markets, International Journal of Industrial Organization, 18, 39-57.

Keser, C., K.-M. Ehrhart, and S. K. Berninghaus, 1998, Coordination and Local Interaction: Experimental Evidence, Economics Letters, 58(3), 269-75.

Kirchkamp, O., 1999, Simultaneous Evolution of Learning Rules and Strategies, Journal of Economic Behavior and Organization, 40(3), 295-312.

— , 2000, Spatial Evolution of Automata in the Prisoners' Dilemma, Journal of Economic Behavior and Organization, 43(2), 239-262.

Kirchkamp, O., and R. Nagel, 2005, Learning and cooperation in network experiments, Discussion Paper 05-27, SFB 504, Universität Mannheim.

Kosfeld, M., 2004, Economic Networks in the Laboratory: A Survey, Review of Network Economics, 3, 20-41.

Lindgreen, K., and M. G. Nordahl, 1994, Evolutionary dynamics of spatial games, Physica $D, 75,292-309$. 
Nowak, M. A., and R. M. May, 1992, Evolutionary Games and Spatial Chaos, Nature, 359, 826-829.

— 1993, The Spatial Dilemmas of Evolution, International Journal of Bifurcation and Chaos, 3, 35-78.

Offerman, T., J. Potters, and J. Sonnemans, 2002, Imitation and Belief Learning in an Oligopoly Experiment, Review of Economic Studies, 69, 973-997.

Offerman, T., and J. Sonnemans, 1998, Learning by experience and learning by imitating successful others, Journal of Economic Behavior and Organization, 34, 559-575.

Pingle, M., and R. H. Day, 1996, Modes of Economizing Behavior: Experimental Evidence, Journal of Economic Behavior and Organization, 29(2), 191-209.

Rogers, W. H., 1993, Regression standard errors in clustered samples, in Stata Technical Bulletin, vol. 13, pp. 19-23. Stata, Reprinted in Stata Technical Bulletins, vol. 3, 88-94.

Selten, R., and J. Apesteguia, 2005, Experimentally observed imitation and cooperation in price competition on the circle, Games and Economic Behavior, 51(1), 171-192.

Selten, R., and A. Ostmann, 2001, Imitation Equilibrium, Homo Oeconomicus, 43, 111149.

Vega-Redondo, F., 1997, The evolution of Walrasian behavior, Econometrica, 65, 375-384.

\section{A List of Sessions}

Overview:

\begin{tabular}{|c|c|c|c|}
\hline \multicolumn{4}{|c|}{ Number of sessions in different treatments } \\
\hline information provided: & detailed & not detailed & $\begin{array}{l}\text { not detailed } \\
\text { computerised } \\
\text { cooperators }\end{array}$ \\
\hline circle, neighbourhood of 5 & 5 & 4 & 5 \\
\hline group, neighbourhood of 5 & 10 & 9 & 0 \\
\hline group, neighbourhood of $8 \ldots 10$ & 5 & 6 & 0 \\
\hline
\end{tabular}


Parameters of each session:

\begin{tabular}{|c|c|c|c|c|c|}
\hline & date & structure & information & $\begin{array}{c}\text { computerised } \\
\text { cooperators }\end{array}$ & $\begin{array}{c}\text { number of } \\
\text { players }\end{array}$ \\
\hline 1 & 19991203111 & group & & & 5 \\
\hline 2 & 19991203112 & group & & & 5 \\
\hline 3 & 19991203141 & group & & & 5 \\
\hline 4 & 19991203142 & group & & & 5 \\
\hline 5 & 19991203143 & group & & & 5 \\
\hline 6 & 19991213131 & group & & & 5 \\
\hline 7 & 19991213132 & group & & & 5 \\
\hline 8 & 19991213133 & group & & & 5 \\
\hline 9 & 19991213134 & group & & & 5 \\
\hline 10 & 20050418-11:05-1 & group & & & 8 \\
\hline 11 & 20050414-18:23-1 & group & & & 9 \\
\hline 12 & 20050414-18:23-2 & group & & & 9 \\
\hline 13 & 20050419-11:15-1 & group & & & 9 \\
\hline 14 & 20050414-16:05-1 & group & & & 10 \\
\hline 15 & 20050414-16:05-2 & group & & & 10 \\
\hline 16 & 19980115-gr1 & group & detailed & & 5 \\
\hline 17 & 19980115-gr2 & group & detailed & & 5 \\
\hline 18 & 19980115-gr3 & group & detailed & & 5 \\
\hline 19 & 19980122-gr1 & group & detailed & & 5 \\
\hline 20 & $19980122-\mathrm{gr} 2$ & group & detailed & & 5 \\
\hline 21 & 19980122-gr3 & group & detailed & & 5 \\
\hline 22 & 19991215131 & group & detailed & & 5 \\
\hline 23 & 19991215132 & group & detailed & & 5 \\
\hline 24 & 19991215133 & group & detailed & & 5 \\
\hline 25 & 19991215134 & group & detailed & & 5 \\
\hline 26 & 20050523-11:43-1 & group & detailed & & 10 \\
\hline 27 & 20050523-11:43-2 & group & detailed & & 10 \\
\hline 28 & 20050523-12:27-1 & group & detailed & & 10 \\
\hline 29 & $20050523-12: 27-2$ & group & detailed & & 10 \\
\hline 30 & 20050524-11:31-1 & group & detailed & & 10 \\
\hline 31 & 1999120113 & circle & & & 14 \\
\hline 32 & 1999120119 & circle & & & 18 \\
\hline
\end{tabular}




\begin{tabular}{|c|l|l|l|c|c|}
\hline \multicolumn{5}{|c|}{ continued from previous page } \\
\hline & date & structure & information & $\begin{array}{c}\text { computerised } \\
\text { cooperators }\end{array}$ & $\begin{array}{c}\text { number of } \\
\text { players }\end{array}$ \\
\hline 33 & 1999120213 & circle & & & 18 \\
34 & 1999120218 & circle & & & 18 \\
35 & $20050415-11: 33$ & circle & & 5 & 13 \\
36 & $20050421-16: 39$ & circle & & 5 & 13 \\
37 & $20050421-18: 21$ & circle & & 5 & 13 \\
38 & $20050426-16: 31$ & circle & & 5 & 13 \\
39 & $20050426-18: 17$ & circle & & 5 & 13 \\
40 & $19980121-c i$ & circle & detailed & & 18 \\
41 & $19980127-c i$ & circle & detailed & & 18 \\
42 & 19980217 -ci & circle & detailed & & 18 \\
43 & 19980218 -ci & circle & detailed & & \\
44 & 1999121315 & circle & detailed & & \\
\hline
\end{tabular}

\section{B Raw data}

In the following tables each line represents the actions of a player from period 1 to period 80 . Cooperation is shown as $\circ$, non cooperation as $\bullet$. Neighbouring lines correspond to neighbouring players in the experiment. In all treatments without computerised cooperators (sections B.7 to B.2) the last line of each block of lines is in circles always a neighbour of the first line of the same block. In these sections the display of circles is always rotated such that least cooperative players are found in the first and the last lines.

\section{B.1 Circle treatment}
$t=1$
20
60
80

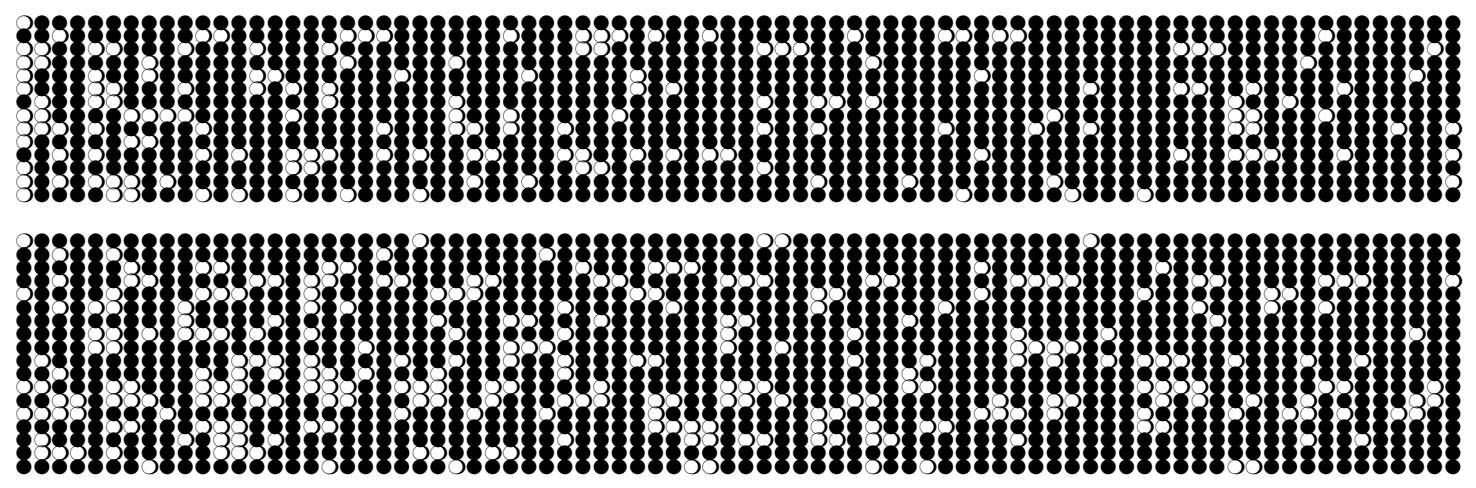



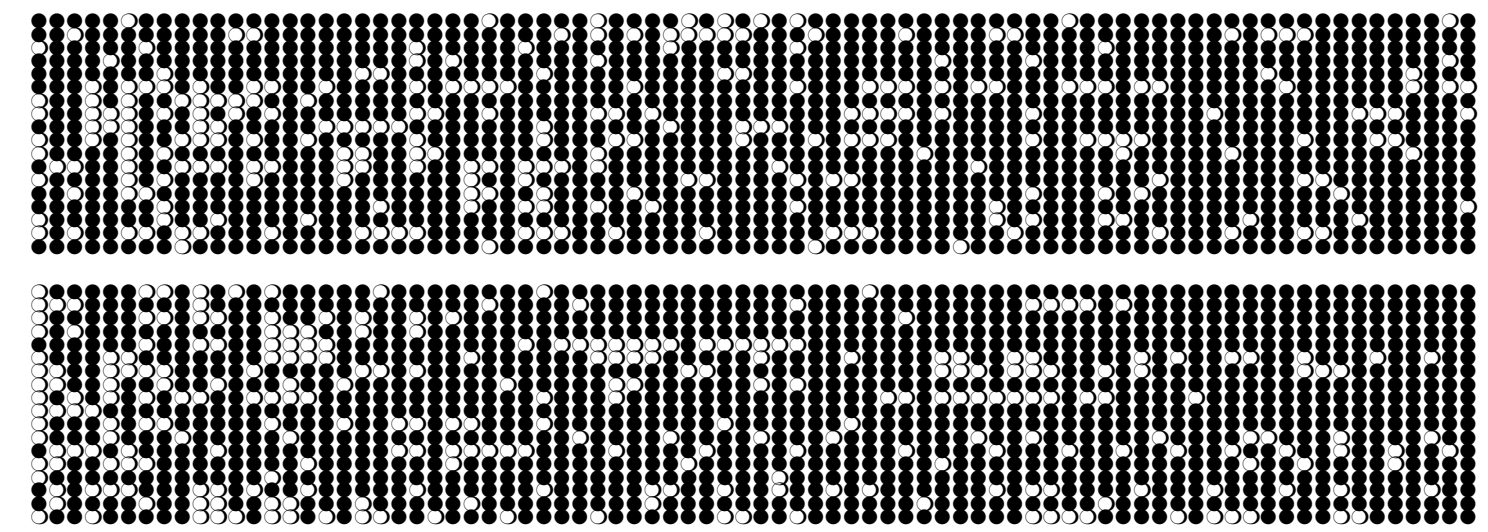

B.2 Group treatment

$t=1$

80

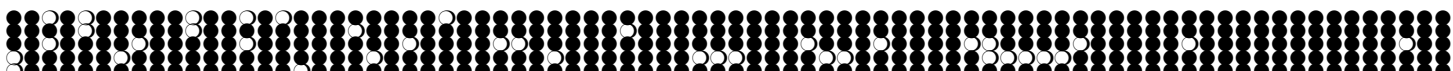

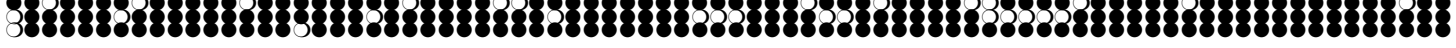

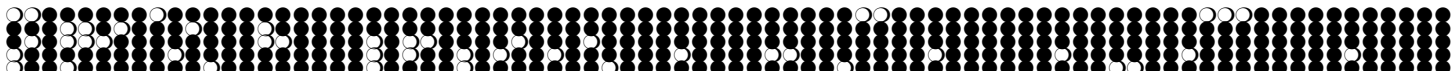

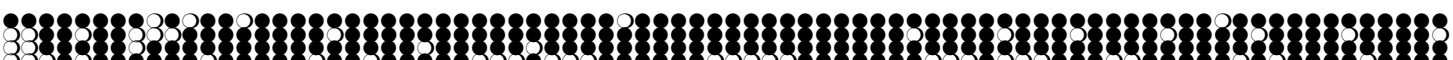

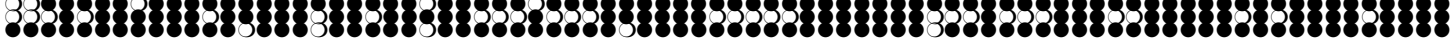
8ร8)

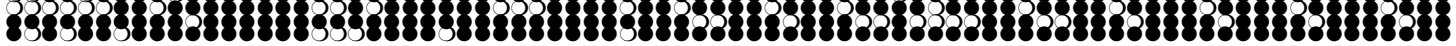

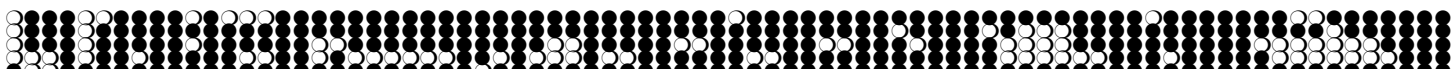

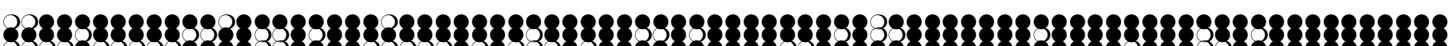

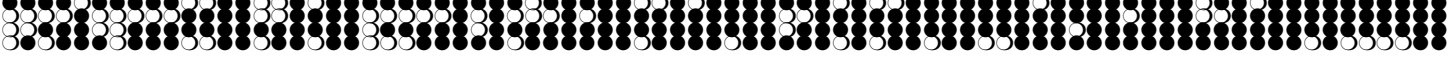

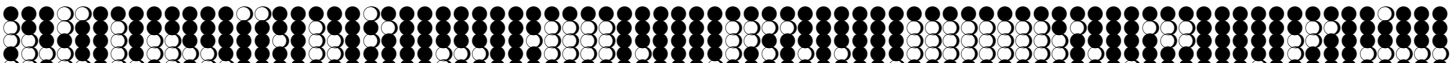

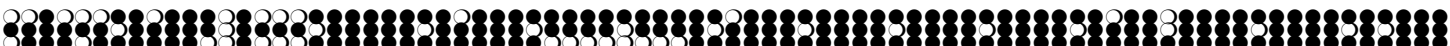

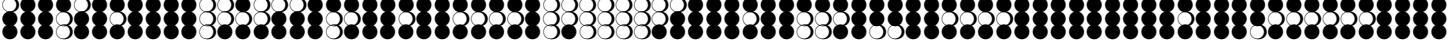

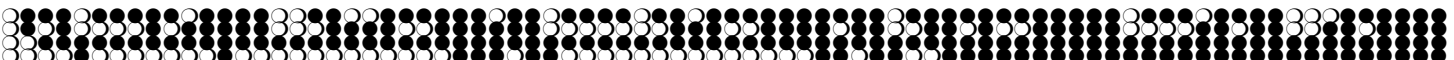

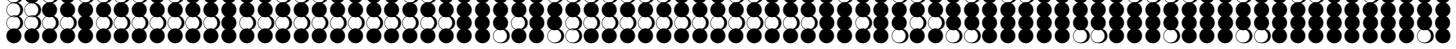

B.3 Groups with a larger neighbourhood

$$
t=1
$$

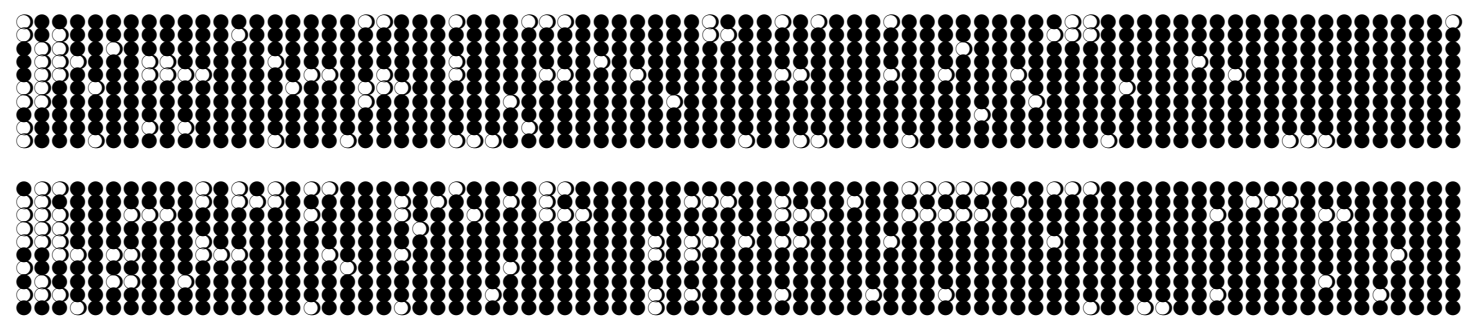




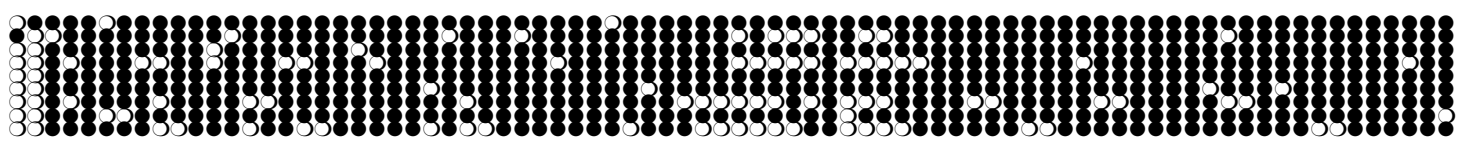

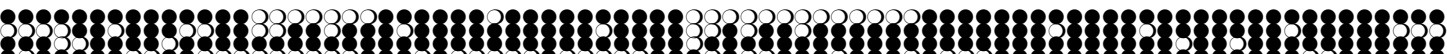

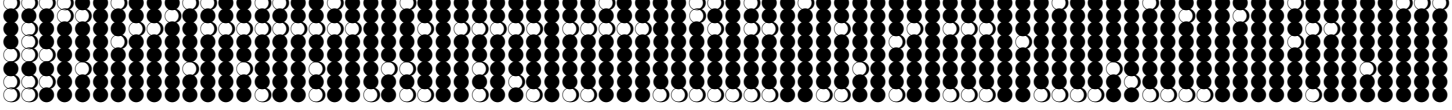
\$2838)

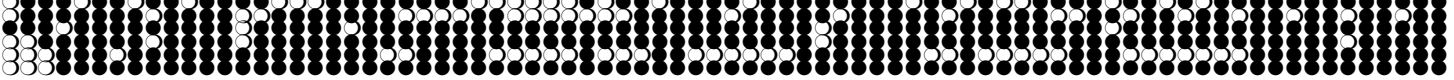

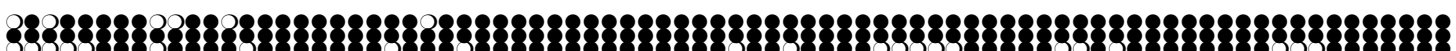

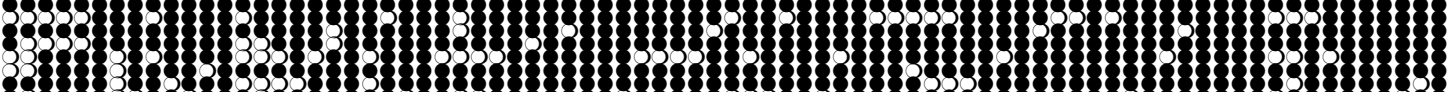

B.4 Group treatment with detailed information

$t=1$

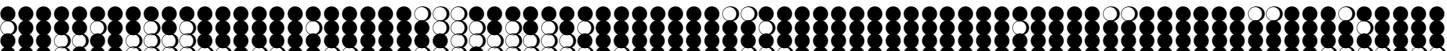

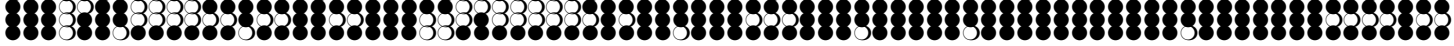

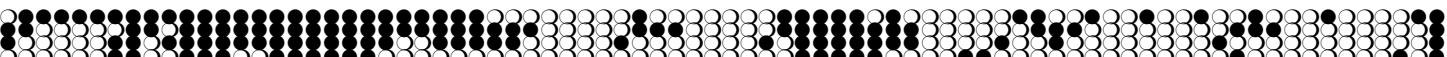

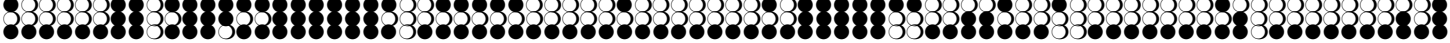

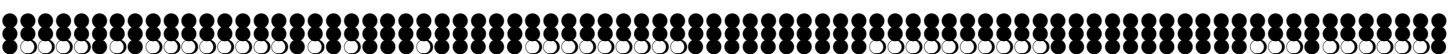

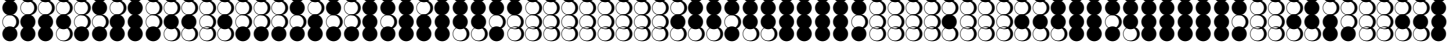

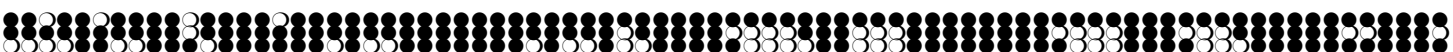

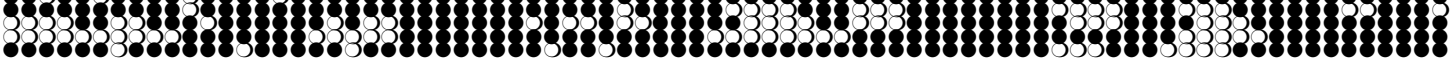

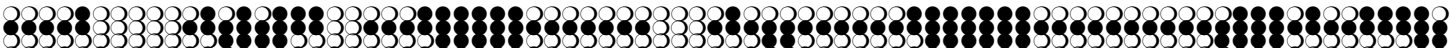
8888

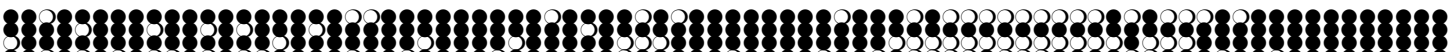

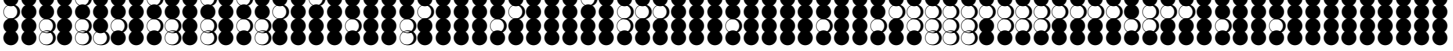

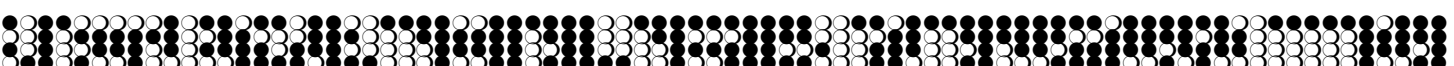
88688 88

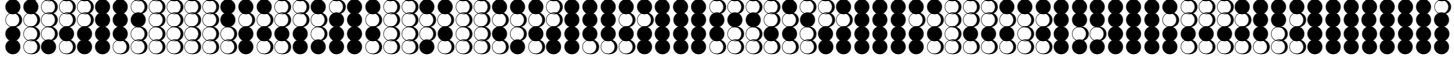

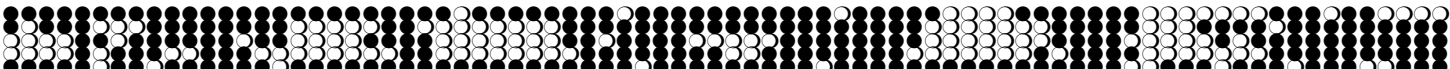

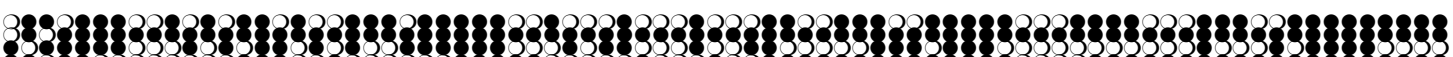

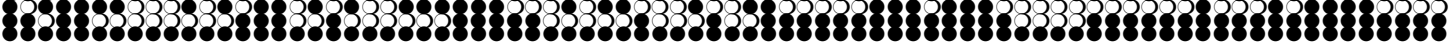

B.5 Group treatment with detailed information and a larger neighbourhood
$t=1$
20
60
80

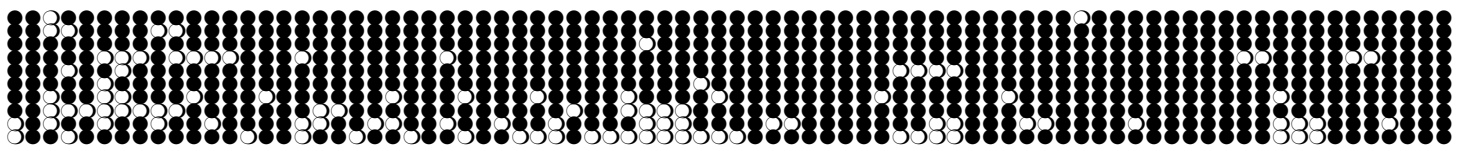



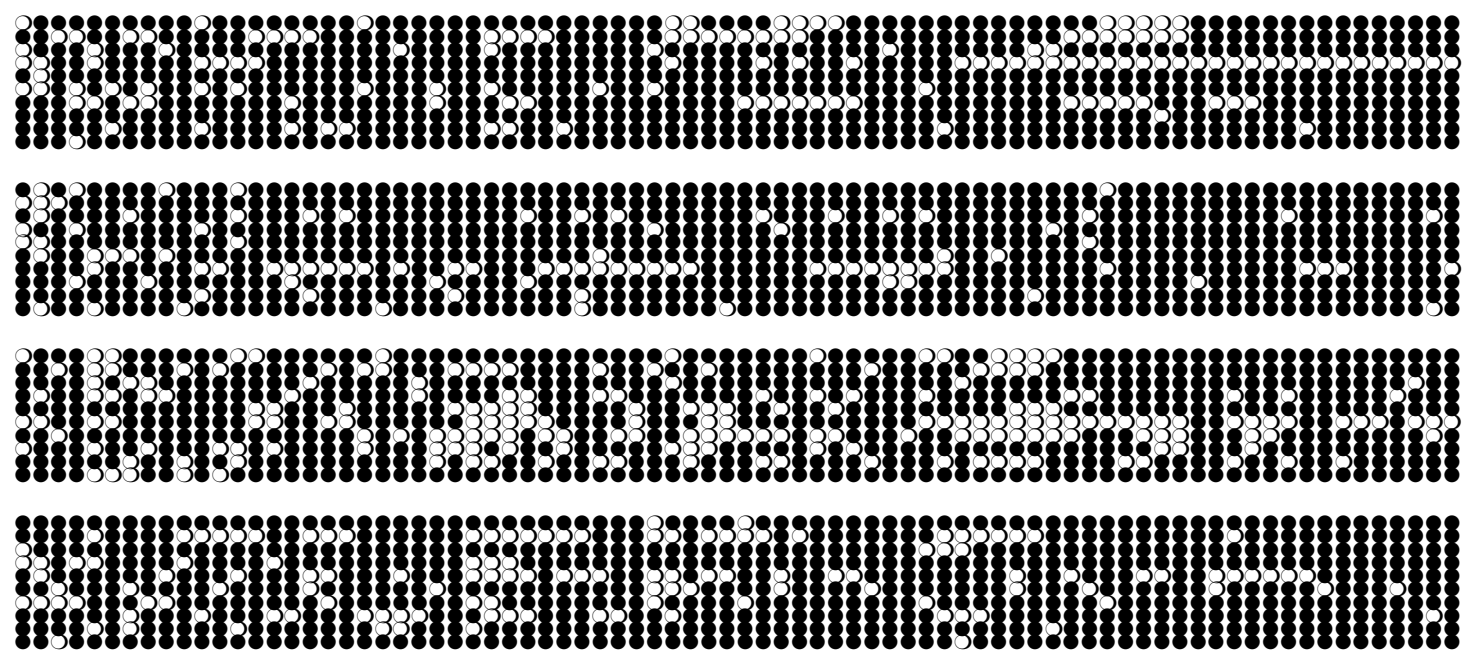

B.6 Circles with some computerised players

$t=1$

60

80
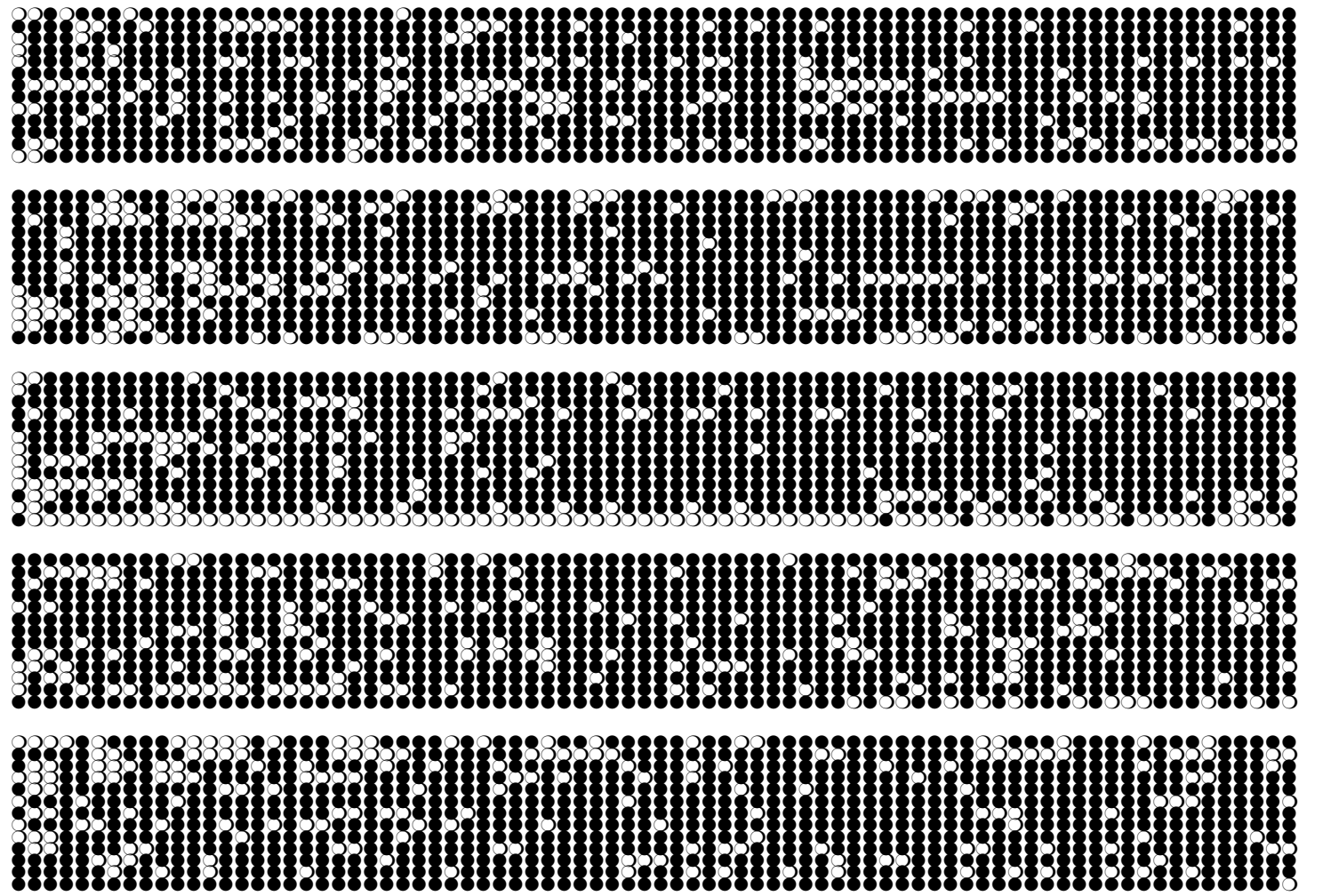

In the display of the circles the five computerised cooperators are not displayed. Their location is on top of the first line and below the last line of each block. The two top lines and the two bottom lines of each block are, hence, immediate neighbours of computerised cooperators. 
B.7 Circle treatment with detailed information

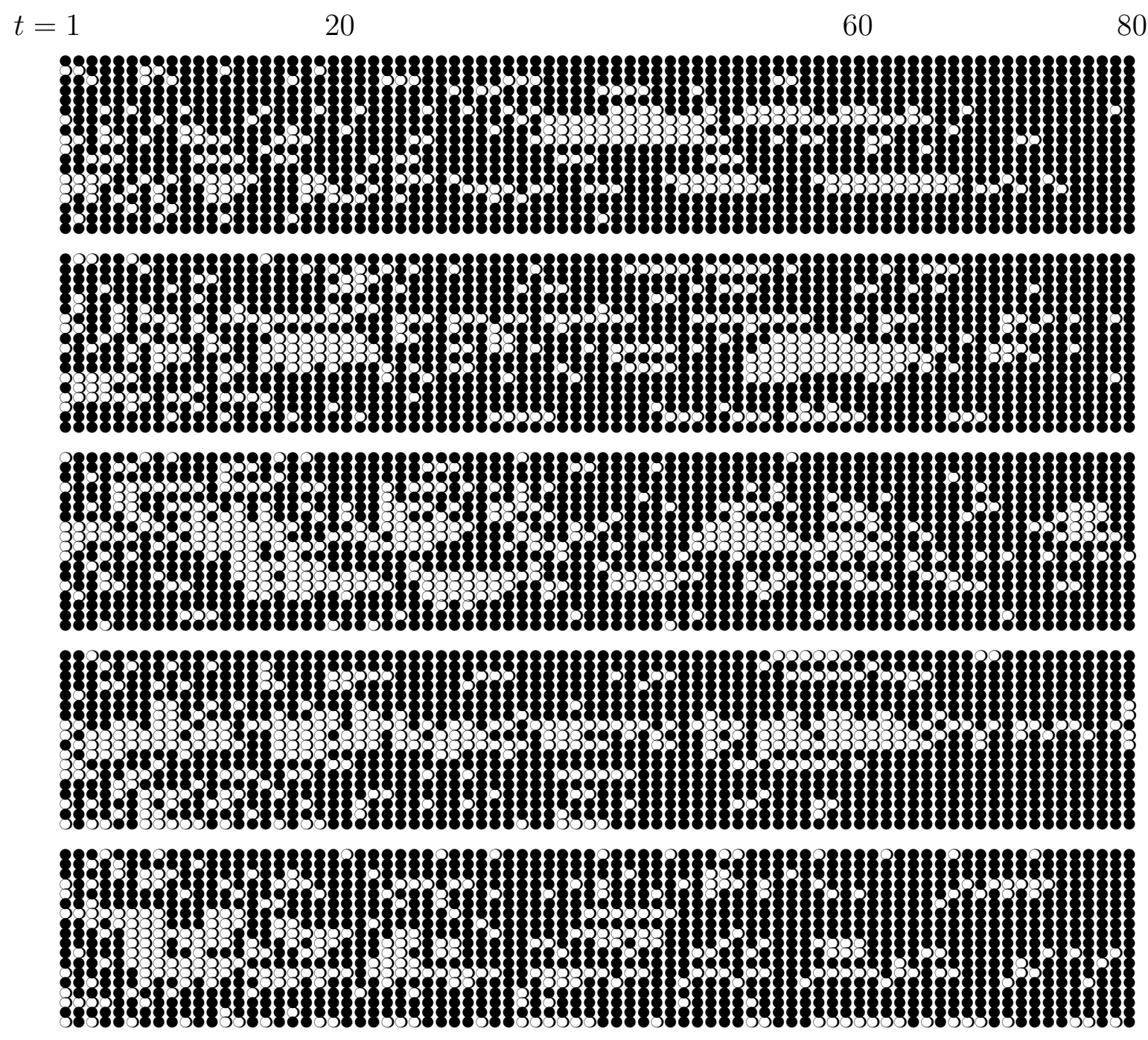

\section{Conducting the experiment and instructions}

All experiments were carried out at Mannheim and Barcelona University. The lab assistants who conducted most of the experiments were not involved in teaching, i.e. not known to participants. ${ }^{18}$ For the group treatments always several groups were present in the lab simultaneously. At the beginning of the experiment participants drew balls from an urn to determine their allocation to seats. Being seated participants then obtained written instructions in German (for the Mannheim experiments) or in Spanish (for the experiments done in Barcelona). The instructions vary slightly depending on the treatment. In the following we give a translation of the instructions.

\footnotetext{
${ }^{18}$ In a few sessions a teacher was present. Behaviour in these sessions was not found to be different from the others.
} 
After answering control questions on the screen subjects entered the treatment described in the instructions. After completing the treatment they answered a short questionnaire on the screen and where then payed in cash.

\section{Instructions}

Please sit down and read the following instructions. It is important that you read them attentively. A good understanding of the game is a prerequisite of your success.

After having read the instructions you will continue with a little quiz on the computer screen. There you will be asked questions that will be easy to answer once you have read the instructions.

You may take notes but you may not talk to each other.

\section{The structure of the neighbourhood}

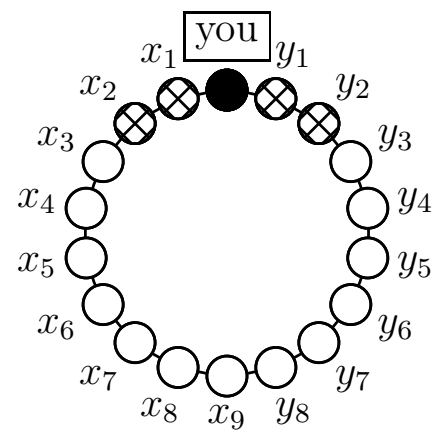

Your gain depends on your decision and on the decision of your two neighbours to the left and your two neighbours to the right. These four neighbours remain the same during the course of the experiment. You are connected through the computer with these neighbours. We will not tell who these neighbours are. Similarly your neighbours will not be told who you are.

In the diagram on the right side your four neighbours are shown cross-hatched. ${ }^{19}$

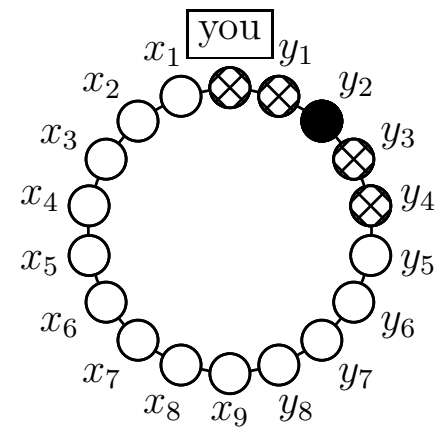

Also your neighbours have neighbours. E.g. the neighbours of $y_{2}$ are players $y_{4}, y_{3}, y_{1}$ and you. ${ }^{20}$

\footnotetext{
${ }^{19}$ The size of the circle shown here and in the figure below depends on the treatment. E.g. in the treatment with groups of five players the circle would be smaller and contain only five players.

${ }^{20}$ In the treatment with groups we say instead: "E.g. the neighbours of $y_{2}$ are players $x_{1}, x_{2}, y_{1}$ and you."

In the treatment with computerised players we add the following: "... In addition to the players that are in a room, five players follow a computerised strategy. Up to two of your neighbours may belong to these players..."
} 


\section{Rounds}

In this experiment you play several rounds. In each round you take a decision. Depending on your decision and on the decision of your neighbours you receive points that will be converted to $€$ at the end of the experiment.

\section{Decision}

In each round you choose among two decisions. You choose A or B. Your gain depends on what you have chosen and on how many of your neighbours have chosen $A$ or $B$.

This relation between choices and gains is the same for all participants. If you choose e.g. A, and all your neighbours choose $B$ then you receive the same number of points as any other person who chooses A while the neighbours of this person all choose B. All players choose simultaneously, without knowing the decisions of the others. ${ }^{21}$

When all players have made their decision we continue with the next round.

\section{Information after each round}

In each round your receive information about your gain. Additionally you receive information about the decisions of your neighbours and their gain.

\begin{tabular}{|c|l|l|l|l|}
\hline Round & Your Decision & Your Gain & $\begin{array}{l}\text { Average gain with } \\
\text { A in your neigh- } \\
\text { bourhood }\end{array}$ & $\begin{array}{l}\text { Average gain with } \\
\text { in your neigh- } \\
\text { bourhood } 22\end{array}$ \\
\hline$\ldots$ & $\ldots$ & $\ldots$ & $\ldots$ & $\ldots$
\end{tabular}

In each row you obtain information about one round. You find your decision and your gain in the second and the third column.

\footnotetext{
${ }^{21}$ In the treatment with detailed information the first part of this paragraph reads instead as follows:

This relation between choices and gains is the same for all participants. It will be shown on the screen in the form of a table.$$
\begin{array}{|c|c|}
\cline { 2 - 2 } \multicolumn{1}{c|}{} & \text { Your neighbours play... } \\
\hline \text { You play A } & \ldots \text {. Your gain ... }
\end{array}
$$

All players choose simultaneously, without knowing the decisions of the others.

${ }^{22}$ In the treatment with detailed information there is only one column with the title: "Decisions and gain in your neighbourhood, ordered by gain"
} 
In the two columns to the right you find the average gain of all players on your neighbourhood who chose $A$ and the average gain for those who chose $B$. The average gain is the sum of gains of all players in the neighbourhood who made a decision divided into the number of these players. Your own gain is included when calculating average gains.

If nobody in the neighbourhood has chosen A or B these columns will be marked with "_.".23

\section{Quiz}

Please answer now the questions from the quiz on the computer screen. If you are unsure how to answer a question, please consult your instructions.

\footnotetext{
${ }^{23}$ In the treatment with detailed information the previous two paragraphs read instead "On the right side we show for each of your neighbours the decision of the neighbour and the obtained gain. The ordering of neighbours in this column depends on the gain in this period. First comes the neighbour with the highest gain, then the one whose gain was second, etc.. This implies that in each period a different person can be the first in the right column."
} 